\title{
Mathematical modelling of cancer invasion: The multiple roles of TGF- $\beta$ pathway on tumour proliferation and cell adhesion
}

\author{
Vasiliki Bitsouni \\ Division of Mathematics, University of Dundee, \\ Dundee, DD1 4HN, Scotland, UK \\ vbitsouni@maths.dundee.ac.uk \\ vbitsouni@gmail.com \\ Mark A. J. Chaplain \\ School of Mathematics and Statistics, \\ Mathematical Institute (MI), North Haugh, \\ University of St Andrews, St Andrews, KY16 9SS, \\ Scotland, UK \\ majc@st-andrews.ac.uk \\ Raluca Eftimie \\ Division of Mathematics, University of Dundee, \\ Dundee, DD1 $4 H N$, Scotland, UK \\ reftimie@maths.dundee.ac.uk \\ Received 9 March 2017 \\ Revised 25 April 2017 \\ Accepted 14 May 2017 \\ Published 6 July 2017 \\ Communicated by N. Bellomo
}

\begin{abstract}
In this paper, we develop a non-local mathematical model describing cancer cell invasion and movement as a result of integrin-controlled cell-cell adhesion and cell-matrix adhesion, and transforming growth factor-beta (TGF- $\beta$ ) effect on cell proliferation and adhesion, for two cancer cell populations with different levels of mutation. The model consists of partial integro-differential equations describing the dynamics of two cancer cell populations, coupled with ordinary differential equations describing the extracellular matrix (ECM) degradation and the production and decay of integrins, and with a parabolic PDE governing the evolution of TGF- $\beta$ concentration. We prove the global
\end{abstract}

This is an Open Access article published by World Scientific Publishing Company. It is distributed under the terms of the Creative Commons Attribution 4.0 (CC-BY) License. Further distribution of this work is permitted, provided the original work is properly cited. 
existence of weak solutions to the model. We then use our model to explore numerically the role of TGF- $\beta$ in cell aggregation and movement.

Keywords: Non-local model of cancer progression; existence; boundedness of solution; cell heterogeneity; TGF- $\beta$; cell-cell and cell-matrix adhesion.

AMS Subject Classification: 35A01, 35Q92, 35R09, 92C15, 92C17, 92-08

\section{Introduction}

Cellular adhesion, i.e. cell--cell and cell-matrix adhesion, and cellular proliferation are fundamental features of multicellular organisms, linked to maintenance of order in the organisms, e.g. tissue formation, stability and breakdown. ${ }^{3}$ These interactions between cells and the extracellular matrix (ECM) are mediated through cell surface receptors, a major group of which is represented by the integrins, 68 and various cytokines and chemokines. Another group of molecules involved in cell--cell adhesion is represented by the cadherin families. ${ }^{26}$ There are several signalling pathways that control normal cell processes like cell proliferation, division, cellular adhesion and apoptosis, with transforming growth factor $\beta$ (TGF- $\beta$ ) pathway to be one of the most critical. Belonging to a large family of multifunctional polypeptides, TGF- $\beta$ regulates the proliferation, differentiation, adhesion, migration and apoptosis of many cell types, including endothelial cells, hematopoietic cells and lymphocytes, $\frac{47}{4}$ and ECM production. 31

Various signals, including integrin, Notch, Wnt, TNF-a, and EGF signals, have been reported to cooperate or synergize with TGF- $\beta$ signalling and stimulate tumour invasion and metastasis. 47 Experimental studies $\$ 44$ showed that the loss of TGF- $\beta$ responsiveness is one of the events that initiate fibrotic disease and malignant progression of cancer, as well as cancer metastasis 65 TGF- $\beta$ induces morphological, biochemical and transcriptional changes towards a mesenchymal phenotype, a process called epithelial to mesenchymal transition (EMT) (see Refs. 42 and 51 and many references therein). EMT occurs when epithelial cells lose their epithelial cell characteristics and become mesenchymal. Mesenchymal cells can return to an epithelial phenotype, a process called mesenchymal-epithelial transition (MET). Through these processes, cancer cells become metastatic and form new colonies at distant sites.

Experimental studies 33141 have shown that tumours consist of heterogeneous populations of cells, which are the result of genetic instability. Intra-tumour heterogeneity appears in almost all phenotypic cell features: from cell morphology, to gene expression, motility, proliferation, immunogenicity and metastatic potential [45 While both normal cells and cancer cells appear to be heterogeneous for various characteristics (e.g. surface antigens), cellular heterogeneity is shown $[\sqrt{54}$ to be more pronounced in malignant neoplasms. Experimental studies have shown complex interactions between clonal cancer cell sub-populations in heterogeneous tumours: from stable coexistence to competitive exclusion $\$ 38$ The metastatic and invasive potential of heterogeneous tumours is influenced by the interactions among the 
cells, and the interactions between cells and ECM components. To detach from the main aggregation/tissue, cells loose cell-cell adhesion and strengthen cell-matrix adhesion (these changes in cell-cell/cell-ECM adhesion can be influenced by TGF$\beta$ signalling), which leads to ECM remodelling and degradation (with the help of enzymes called matrix metalloproteinases; MMPs).

Over the last three decades there have been multiple mathematical models introduced to investigate the formation and movement of various cell aggregations (see, for example, Refs. 1 [3, 5, 6, 8, 48, 49] 55, 56] and 59, and the many references therein). While there are mathematical models in the literature that investigate the roles of TGF- $\beta$ on cancer dynamics, generally these models focus on particular aspects of cancer progression (e.g. growth 4 46163). There are very few models that investigate, in an integrated manner, the multiple roles of TGF- $\beta$ on cancer evolution (see, for example Refs. 4 and 67), and in general these models focus on the motility and growth rate of early stage cancer cells.

In this study, we present a novel mathematical model which investigates in a integrated manner the various roles of TGF- $\beta$ on tumour growth/decay, and on cellcell and cell-matrix interactions, but paying particular attention to the opposite role of TGF- $\beta$ on early stage versus late stage cancer cells. To this end, we introduce a non-local hyperbolic-parabolic model for cell-cell and cell-matrix adhesion for two cancer cell populations: an early stage cancer population, moving both randomly and in a directed manner in response to cell-cell and cell-matrix adhesive forces, and a late stage cancer population (i.e. a mutated clone) moving only in a directed manner following self-adhesive and cross-adhesive cell-cell forces, as well as matrix interactions. Since TGF- $\beta$ does not affect only tumour growth, but impacts also cell adhesion,, we model the interactions between TGF- $\beta$ and integrins that influence the cell-cell and cell-matrix adhesive forces. The computational results show a range of heterogeneous invasion patterns, as a result of the opposite role of TGF- $\beta$ in early and late stages of cancer. Analytical results show the global existence of bounded solutions (hence existence of various types of invasion patterns). We note that existence results have been shown for local nonlinear parabolic PDEs for cell movement coupled with ODEs describing the ECM dynamics with tissue remodelling, 22]326162 as well as for non-local parabolic models describing cancer invasion when the ECM production is zero ${ }^{10}$ and when it is nonzero $\frac{60}{}$ Existence results have also been shown for local hyperbolic models for chemo-sensitive movement 29 However, in contrast to these previous results, here we show existence for a non-local parabolic-hyperbolic model for cancer cell movement.

The structure of this paper is as follows. In Sec. 2 we present our mathematical model, which consists of partial integro-differential equations describing the dynamics of cell populations in early and late stages of cancer, coupled with ordinary differential equations describing ECM and integrins dynamics, and a parabolic partial differential equation describing TGF- $\beta$ dynamics. In Sec. 3, we present a suitable notion of weak solution to the model and we prove the global-in-time existence 
of bounded solutions to our system as the vanishing viscosity limit of a classical solution to an associated parabolic problem. In Sec.4 we undertake numerical simulations to investigate the effect of TGF- $\beta$ on cancer cell movement, and observe a range of patterns obtained for different values of parameters of the model. Finally, in Sec. 5. we summarise our results and give some concluding remarks.

\section{The Mathematical Model of TGF- $\beta$ Regulatory Network in Cancer}

TGF- $\beta$ plays a crucial role in embryonic development, wound healing and cancer. Moreover, TGF- $\beta$ signalling stimulates EMT in certain epithelial cells (see Ref. 51 and many references therein) and consequently induces various diseases, including cancer. The way that TGF- $\beta$ interacts with cancer cells varies between early and late stages of cancer (see Fig. 1), making its behaviour difficult to analyse. We consider a two-population model describing the behaviour of an early stage cancer population and a late stage cancer (descendant clone) population, which interact with each other, as well as with the ECM, via long-range integrin-controlled adhesive and repulsive forces ${ }^{18 \mid 23}$ on bounded spatial domain $\Omega \subset \mathbb{R}^{n}$ with smooth boundary $\partial \Omega$. For $T>0$ let $\Omega_{T}=(0, T) \times \Omega$. Denote by $u_{1}(t, x)$ and $u_{2}(t, x)$ the density of early and late stage cancer cells, respectively, at position $x$ and time $t$, by $f(t, x)$ the ECM density, and by $c(t, x)$ the density of integrin receptors on the surface of cancer cells (receptors involved in cell-cell and cell-matrix interactions). Finally, we denote by $b(t, x)$ the TGF- $\beta$ concentration. For compact notation, we define the vectors $\underline{u}(t, x)=\left(u_{1}(t, x), u_{2}(t, x)\right)^{\top}$ and $\underline{v}(t, x)=(\underline{u}(t, x), f(t, x))^{\top}$.

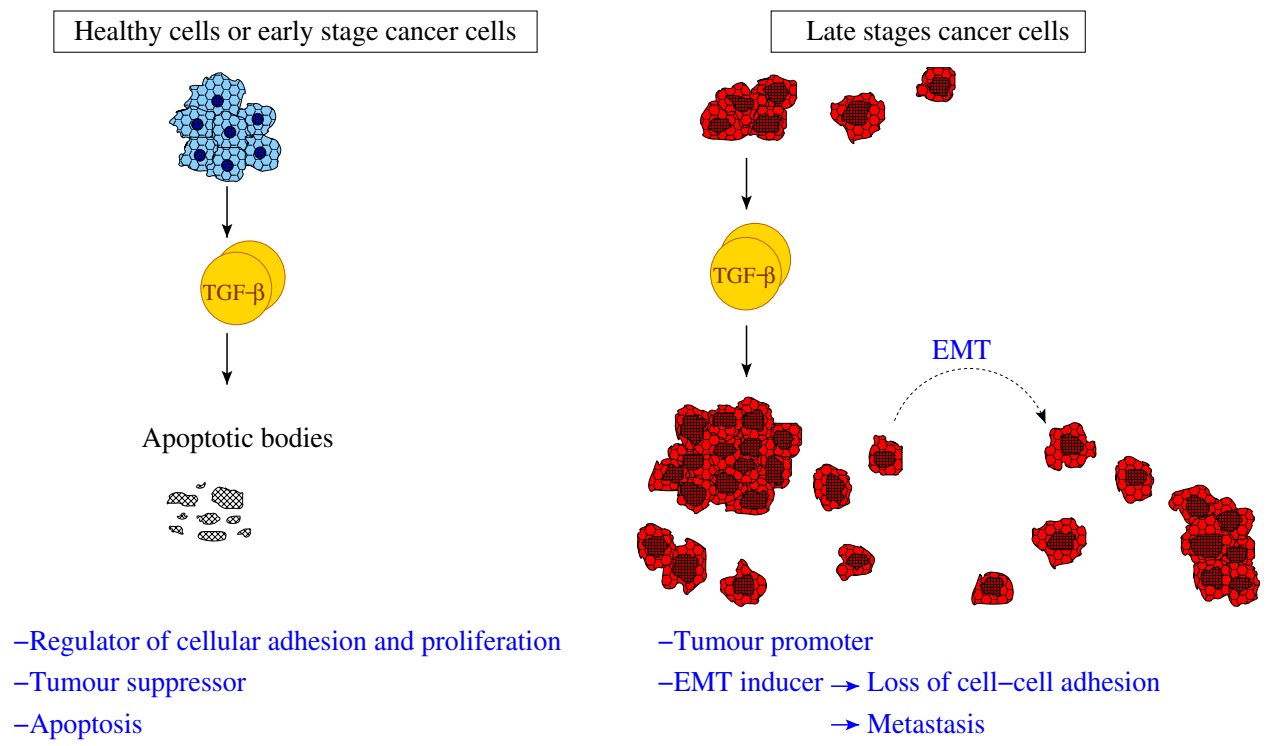

Fig. 1. A caricature summarising the dual role of TGF- $\beta$ in cancer progression. 
Cancer cells dynamics. Cancer cells can switch from a homogeneous type of invasion to a heterogeneous type of invasion described by (directionally moving) invading chains. ${ }^{12}$ Therefore, we assume that the movement of the early stage cancer cell population $u_{1}$ is governed by random motility (which underlines a homogeneous type of invasion), as well as directed motility in response to cell-cell and cell-matrix adhesive forces (which underlines the heterogeneous type of invasion) $\frac{9}{9}$ Let $D_{u}$ describe the random motility coefficient and $F_{1}[\underline{u}, f, c, b]$ describe the nonlocal directed motility. In contrast, the late stage cancer cell population, $u_{2}$, moves only in a directed manner (hence exhibiting a heterogeneous type of invasion) in response to cell--cell and cell-matrix adhesion forces (described by a non-local term $\left.F_{2}[\underline{u}, f, c, b]\right)$. Moreover, the $u_{1}$ cells can mutate into $u_{2}$ cells at a constant rate $M$. TGF- $\beta$ has been found to have bidirectional functions in the progression of cancer. In early stages of cancer, TGF- $\beta$ is an antiproliferative and proapoptotic signal, while in late stages of cancer it acts as a tumour promoter. $\underline{57}$ Thus, we have the following equations describing the dynamics of the two cancer cell populations:

$$
\begin{aligned}
& \frac{\partial u_{1}}{\partial t}=D_{u} \Delta u_{1}-\nabla \cdot\left(u_{1} F_{1}[\underline{u}, f, c, b]\right)-M u_{1}+G_{1}(\underline{u}, b), \\
& \frac{\partial u_{2}}{\partial t}=-\nabla \cdot\left(u_{2} F_{2}[\underline{u}, f, c, b]\right)+M u_{1}+G_{2}(\underline{u}, b) .
\end{aligned}
$$

Taking into account the effect of TGF- $\beta$ on cancer cell proliferation and assuming that both $u_{1}$ and $u_{2}$ cells can proliferate in a logistic manner (to describe the observed slow-down in tumour growth following the loss of nutrients 37 ), we choose the growth functions to be given by

$$
G_{i}(\underline{u}, b)=r_{i} u_{i}\left(1-\frac{u_{1}+u_{2}}{k_{u}}\right)\left(1+(-1)^{i} c_{b} \frac{b}{b_{m}}\right), \quad i=1,2,
$$

where $r_{1}$ and $r_{2}$ are the growth rates of the $u_{1}$ and $u_{2}$ populations, respectively, $k_{u}$ is the carrying capacity, $b_{m}$ is the maximum TGF- $\beta$ concentration, and $c_{b}$ is a coefficient related to the effect of TGF- $\beta$ on cancer cell proliferation/decay. In particular, the term $(-1)^{i}$ models the anti-tumour effect of TGF- $\beta$ on early tumours $(i=1)$, and the pro-tumour effect on late tumours $(i=2)$. Note that these growth functions incorporate also the principle of competition between clonal sub-populations in heterogeneous tumours. $\stackrel{[38}{[3}$

The non-local cell-cell and cell-matrix adhesion and repulsion forces for cancer cell populations $u_{1}$ and $u_{2}$, are described by a function that depends on cell densities, ECM and integrin densities, and concentrations of TGF- $\beta$ molecules

$$
F_{i}: C(\bar{\Omega}: \mathbb{R})^{5} \mapsto C^{1, \zeta}\left(\bar{\Omega}: \mathbb{R}^{n}\right), \quad \zeta \in(0,1], \quad i=1,2,
$$

given by the following relation

$$
F_{i}[\underline{u}, f, c, b](x):=\int_{\Omega} K(|y-x|) g_{i}(\underline{u}(y), f(y), c(x), b(x)) \mathrm{d} y, \quad i=1,2,
$$

where $K \in L^{\infty}(\Omega)$, with $\partial_{x} K \in L^{\infty}(\Omega)$. The functions $g_{i}(\underline{u}, f, c, b), i=1,2$, describe the nature of the cell-cell and cell-matrix adhesive forces. These functions increase 
when the cell density and ECM density increase, and accordingly they decrease when the cell density and ECM density decrease. The functions $g_{i}, i=1,2$, are given by

$$
g_{i}(\underline{u}, f, c, b):=S_{i}(c, b) u_{i}+S(c, b) u_{j}+C_{i}(c, b) f, \quad i, j=1,2, i \neq j,
$$

where $S_{i}(c, b)$ is the cell-cell self-adhesion strength function for populations $u_{i}$, $S(c, b)$ is the cell-cell cross-adhesion strength function between the two populations, and $C_{i}(c, b)$ is the adhesion strength function between population $u_{i}$ and ECM.

Integrins are molecules known to have a regulative role in cell-cell and cellmatrix adhesion, $\stackrel{4}{,}$ while the role of TGF- $\beta$ in cellular adhesion is dual: (i) Promotes cell-matrix adhesion by inducing the synthesis and the secretion of ECM-adhesion molecules laminin and fibronectin and the upregulation of integrin expression for these matrix-adhesion molecules ${ }^{3066}$, (ii) Decreases cell-cell adhesion. $52[67]$ Thus, to define these adhesion strength functions we consider the integrin density, $c$, and TGF- $\beta$ concentration, $b$. Since cell mutation could lead to more integrins,, 35 we consider strength functions with different integrin levels for each of the two populations. The more integrins a cell has, the stronger its adhesion force. Therefore, biologically realistic choices for these adhesion strength functions are the increasing, bounded and positive functions given by:

$$
\begin{aligned}
& S_{i}(c, b)=s_{i}{ }^{*}\left(1+\tanh \left(a_{i} c-a_{b_{i}} b\right)\right), \quad S(c, b)=s^{*}\left(1+\tanh \left(d c-d_{b} b\right)\right), \\
& C_{i}(c, b)=c_{i}{ }^{*}\left(1+\tanh \left(e_{i} c+e_{b_{i}} b\right)\right), \quad i=1,2,
\end{aligned}
$$

where $a_{i}, a_{b_{i}}, d, d_{b}, e_{i}, e_{b_{i}}$ and $s_{i}{ }^{*}, s^{*}, c_{i}{ }^{*}, i=1,2$, are positive real numbers.

ECM dynamics. The ECM is considered as non-motile matter, with changes to its density due to degradation by $u_{1}$ and $u_{2}$ cell populations upon contact at rates $\alpha>0$ and $\beta>0$, respectively, and ECM density remodelling back to normal levels, at a constant rate of $\delta>0$. Moreover, TGF- $\beta$ induces the synthesis of ECM

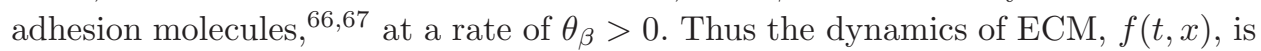
described by:

$$
\frac{\partial f}{\partial t}=-\alpha u_{1} f-\beta u_{2} f+\theta_{\beta} b f+\delta f\left(1-\frac{f}{f_{m}}\right),
$$

where $f_{m}$ is the maximum ECM density at which the ECM fills up all available physical space.

Integrin dynamics. We assume that the level of integrins depends on cancer cell density, such that cell mutation changes the density of receptors (since in highly metastatic cancers, the expression of integrins is up-regulated $\sqrt{35}$ ). Moreover, TGF- $\beta$ signalling up-regulates the integrin expression, ${ }^{442}$ at a rate of $p_{3}$. Therefore, the dynamics of integrins, $c(t, x)$, can be described by:

$$
\frac{\partial c}{\partial t}=p_{1} u_{1}+p_{2} u_{2}+p_{3} b c-q c
$$


where $q$ is the decay rate of $c$, and $p_{1}$ and $p_{2}$ are the production rates of integrins by $u_{1}$ and $u_{2}$ cancer cell populations, respectively. To model the increase in receptors on highly mutated cancer cells, we assume that $p_{2}>p_{1}$ (see Table A.2).

TGF- $\boldsymbol{\beta}$ dynamics. Finally, TGF- $\beta$ is assumed to diffuse freely in the spatial domain, after being released by $u_{1}$ and $u_{2}$ cells, and decay at a rate of $q_{b}>0$. Therefore, the dynamics of TGF- $\beta, b(t, x)$, is described by:

$$
\frac{\partial b}{\partial t}=D_{b} \Delta b+\lambda(\underline{u})-q_{b} b
$$

where $D_{b}$ is the TGF- $\beta$ diffusion coefficient and $\lambda(\underline{u})$ is the TGF- $\beta$ production term. Here, we choose $\lambda(\underline{u})=\mu_{1} u_{1}+\mu_{2} u_{2}$, with $\mu_{1}$ and $\mu_{2}$ to be the production rates of TGF- $\beta$ by $u_{1}$ and $u_{2}$, respectively.

The relations 2.1 -2.9 are summarised in the following system:

$$
\begin{aligned}
\frac{\partial u_{1}}{\partial t} & =D_{u} \Delta u_{1}-\nabla \cdot\left(u_{1} F_{1}[\underline{u}, f, c, b]\right)-M u_{1}+G_{1}(\underline{u}, b), \\
\frac{\partial u_{2}}{\partial t} & =-\nabla \cdot\left(u_{2} F_{2}[\underline{u}, f, c, b]\right)+M u_{1}+G_{2}(\underline{u}, b), \\
\frac{\partial f}{\partial t} & =-\alpha u_{1} f-\beta u_{2} f+\theta_{\beta} b f+\delta f\left(1-\frac{f}{f_{m}}\right), \\
\frac{\partial c}{\partial t} & =p_{1} u_{1}+p_{2} u_{2}+p_{3} b c-q c \\
\frac{\partial b}{\partial t} & =D_{b} \Delta b+\mu_{1} u_{1}+\mu_{2} u_{2}-q_{b} b .
\end{aligned}
$$

We impose the following initial conditions:

$$
\begin{gathered}
u_{i}(0, x)=u_{i_{0}}(x) \geq 0, \quad i=1,2, \quad f(0, x)=f_{0}(x) \geq 0, \\
c(0, x)=c_{0}(x) \geq 0, \quad b(0, x)=b_{0}(x) \geq 0, \quad \text { in } \Omega .
\end{gathered}
$$

Finally, we assume that there is no-flux of both cancer cells and TGF- $\beta$ proteins on the boundary of the domain,

$$
\left\langle\nabla u_{i}, \nu\right\rangle=0=\langle\nabla b, \nu\rangle, \quad i=1,2, \quad \text { on }(0, \infty) \times \partial \Omega
$$

and

$$
\left\langle F_{i}[\underline{u}, f, c, b], \nu\right\rangle=0, \quad i=1,2, \quad \text { on }(0, \infty) \times \partial \Omega,
$$

where $\nu$ is the outward unit normal vector to $\partial \Omega$.

\section{Existence of Solution}

To prove the existence of solution for system (2.10), we use the theory of semigroups combined with the vanishing viscosity method (to transform Eq. 2.10b) into a parabolic equation). Then we show that in the vanishing viscosity limit, we obtain weak solutions for (2.10). We note that the steps in this proof of existence 
of approximate solution follow similar approaches taken in Ref. 10, where a simpler parabolic-ODE model with no production term in the ODE (i.e. the ODE in Ref. 10 contains only decay term, which implies global boundedness of its solution) is considered, whereas in our model the production terms in (2.10d)-(2.10d) add an extra layer of complexity to the proof. Moreover, for the proof of vanishing viscosity limit we use techiques similar to those in Ref. 29] for a hyperbolic-elliptic model. The link between these two proofs is based on the extraction of the appropriate estimates for the vanishing viscosity limit, for our more complex system of non-local parabolic-hyperbolic equations coupled with ODEs.

\subsection{Existence of approximate solution}

We will approximate system (2.10) by the following system:

$$
\begin{aligned}
\frac{\partial u_{1}^{\epsilon}}{\partial t}-D_{u} \Delta u_{1}^{\epsilon}+M u_{1}^{\epsilon}= & -\nabla u_{1}^{\epsilon} \cdot F_{1}\left[u_{1}^{\epsilon}, u_{2}^{\epsilon}, f^{\epsilon}, c^{\epsilon}, b^{\epsilon}\right] \\
& -u_{1}^{\epsilon}\left(\nabla \cdot F_{1}\left[u_{1}^{\epsilon}, u_{2}^{\epsilon}, f^{\epsilon}, c^{\epsilon}, b^{\epsilon}\right]\right)+G_{1}\left(u_{1}^{\epsilon}, u_{2}^{\epsilon}, b^{\epsilon}\right), \\
\frac{\partial u_{2}^{\epsilon}}{\partial t}-\epsilon \Delta u_{2}^{\epsilon}+u_{2}^{\epsilon}= & -\nabla u_{2}^{\epsilon} \cdot F_{2}\left[u_{1}^{\epsilon}, u_{2}^{\epsilon}, f^{\epsilon}, c^{\epsilon}, b^{\epsilon}\right] \\
& -u_{2}^{\epsilon}\left(\nabla \cdot F_{2}\left[u_{1}^{\epsilon}, u_{2}^{\epsilon}, f^{\epsilon}, c^{\epsilon}, b^{\epsilon}\right]\right)+h_{1}\left(u_{1}^{\epsilon}, u_{2}^{\epsilon}, b^{\epsilon}\right), \\
\frac{\partial f^{\epsilon}}{\partial t}= & h_{2}\left(u_{1}^{\epsilon}, u_{2}^{\epsilon}, b^{\epsilon}\right) f^{\epsilon}-\frac{\delta}{f_{m}} f^{\epsilon^{2}}, \\
\frac{\partial c^{\epsilon}}{\partial t}= & h_{3}\left(u_{1}^{\epsilon}, u_{2}^{\epsilon}\right)+c^{\epsilon} h_{4}\left(b^{\epsilon}\right), \\
\frac{\partial b^{\epsilon}}{\partial t}-D_{b} \Delta b^{\epsilon}+q_{b} b^{\epsilon}= & h_{5}\left(u_{1}^{\epsilon}, u_{2}^{\epsilon}\right),
\end{aligned}
$$

for $0<\epsilon \leq 1$, where

$$
\begin{aligned}
h_{1}\left(u_{1}^{\epsilon}, u_{2}^{\epsilon}, b^{\epsilon}\right) & =M u_{1}^{\epsilon}+u_{2}^{\epsilon}+G_{2}\left(u_{1}^{\epsilon}, u_{2}^{\epsilon}, b^{\epsilon}\right), \\
h_{2}\left(u_{1}^{\epsilon}, u_{2}^{\epsilon}, b^{\epsilon}\right) & =-\alpha u_{1}^{\epsilon}-\beta u_{2}^{\epsilon}+\theta_{\beta} b^{\epsilon}+\delta, \\
h_{3}\left(u_{1}^{\epsilon}, u_{2}^{\epsilon}\right) & =p_{1} u_{1}^{\epsilon}+p_{2} u_{2}^{\epsilon}, \quad h_{4}\left(b^{\epsilon}\right)=p_{3} b^{\epsilon}-q \quad \text { and } \\
h_{5}\left(u_{1}^{\epsilon}, u_{2}^{\epsilon}\right) & =\mu_{1} u_{1}^{\epsilon}+\mu_{2} u_{2}^{\epsilon} .
\end{aligned}
$$

The ICs are given by

$$
\begin{aligned}
& u_{i}^{\epsilon}(0, x)=u_{i_{0}}(x) \geq 0, \quad i=1,2, \quad f^{\epsilon}(0, x)=f_{0}(x) \geq 0, \\
& c^{\epsilon}(0, x)=c_{0}(x) \geq 0, \quad b^{\epsilon}(0, x)=b_{0}(x) \geq 0, \quad \text { in } \Omega .
\end{aligned}
$$

Finally, the BCs corresponding to 2.12 - 2.13 are given by the relations

$$
\left\langle\nabla u_{i}^{\epsilon}, \nu\right\rangle=0=\left\langle\nabla b^{\epsilon}, \nu\right\rangle, \quad i=1,2, \quad \text { on }(0, \infty) \times \partial \Omega
$$

and

$$
\left\langle F_{i}\left[u_{1}^{\epsilon}, u_{2}^{\epsilon}, f^{\epsilon}, c^{\epsilon}, b^{\epsilon}\right], \nu\right\rangle=0, \quad i=1,2, \quad \text { on }(0, \infty) \times \partial \Omega \text {. }
$$


For the full non-local interaction terms 2.3 -2.4 we make the following assumptions:

$$
F_{i}\left[u_{1}, u_{2}, f, c, b\right](x)=\int_{\Omega} N_{i}\left(x, y, u_{1}(y), u_{2}(y), f(y), c(x), b(x)\right) \mathrm{d} y,
$$

where $N_{i}: \Omega^{2} \times \mathbb{R}^{5} \mapsto \mathbb{R}^{n}, i=1,2$, is a continuous function, which satisfies

$$
N_{i}(x, y, \underline{0}, c, b)=0, \quad i=1,2, \quad \text { for all }(x, y) \in \Omega^{2}, c, b \in \mathbb{R},
$$

and

$$
N_{i}(\cdot, y, \phi, \chi, \psi, c, b) \in C^{1, \zeta}\left(\bar{\Omega}: \mathbb{R}^{n}\right), \quad i=1,2, \quad \text { for all } y \in \Omega, \quad(\phi, \chi, \psi) \in \mathbb{R}^{3} .
$$

Since functions $S_{i}(c, b), S(c, b), C_{i}(c, b), i=1,2$, (given by 2.6) are bounded, we assume that there is a constant $L_{N}$, which depends on the bound for $S_{i}, S, C_{i}, i=$ 1,2 , such that for any $\phi_{1}, \phi_{2}, \chi_{1}, \chi_{2}, \psi_{1}, \psi_{2} \in \mathbb{R}$ we have

$$
\begin{aligned}
& \left|N_{i}\left(x, y, \phi_{1}, \chi_{1}, \psi_{1}, c, b\right)-N_{i}\left(x, y, \phi_{2}, \chi_{2}, \psi_{2}, c, b\right)\right| \\
& \quad+\left|\partial_{x} N_{i}\left(x, y, \phi_{1}, \chi_{1}, \psi_{1}, c, b\right)-\partial_{x} N_{i}\left(x, y, \phi_{2}, \chi_{2}, \psi_{2}, c, b\right)\right| \\
& \leq L_{N}\left(\left|\phi_{1}-\phi_{2}\right|+\left|\chi_{1}-\chi_{2}\right|+\left|\psi_{1}-\psi_{2}\right|\right), \quad i=1,2,
\end{aligned}
$$

uniformly with respect to $(x, y) \in \Omega^{2}$.

We assume that $h_{2}^{\prime}, h_{3}^{\prime}$ and $h_{4}^{\prime}$ exist, and that

$$
h_{2}^{\prime}: \mathbb{R} \times \mathbb{R} \times \mathbb{R} \mapsto \mathbb{R}, \quad h_{3}^{\prime}: \mathbb{R} \times \mathbb{R} \mapsto \mathbb{R} \quad \text { and } \quad h_{4}^{\prime}: \mathbb{R} \mapsto \mathbb{R}
$$

are locally Lipschitz functions.

Based on relation (2.2) we can assume that there are constants $B_{i}, D_{i}>0, i=$ 1,2 , such that for $u_{1}^{\epsilon}, u_{2}^{\epsilon}, b^{\epsilon} \geq 0, i=1,2$, we have

$$
G_{i}\left(u_{1}^{\epsilon}, u_{2}^{\epsilon}, b^{\epsilon}\right) \leq B_{i}-D_{i} u_{i}^{\epsilon}, \quad i=1,2 .
$$

Moreover, based on relation (3.2) we can assume that there are constants $\Lambda_{j}>$ $0, j=1, \ldots, 4$, such that for $u_{1}^{\epsilon}, u_{2}^{\epsilon}, f^{\epsilon}, c^{\epsilon}, b^{\epsilon} \geq 0$ :

$$
f^{\epsilon} h_{2}\left(u_{1}^{\epsilon}, u_{2}^{\epsilon}, b^{\epsilon}\right)-\frac{\delta}{f_{m}} f^{\epsilon^{2}} \leq \Lambda_{1} b^{\epsilon}-\Lambda_{2} f^{\epsilon}
$$

and

$$
c^{\epsilon} h_{4}\left(b^{\epsilon}\right) \leq \Lambda_{3} b^{\epsilon}-\Lambda_{4} c^{\epsilon} .
$$

We now consider the sectorial operators

$$
A_{1}=-D_{u} \Delta+M I, \quad A_{2}=-\epsilon \Delta+I \quad \text { and } \quad A_{3}=-D_{b} \Delta+q_{b} I
$$

in the space $X=L^{p}(\Omega)$, with common domain of definition

$$
D=D\left(A_{1}\right)=D\left(A_{2}\right)=D\left(A_{3}\right)=\left\{w \in W^{2, p}: \frac{\partial w}{\partial \nu}=0 \text { on } \partial \Omega\right\},
$$


and $\operatorname{Re}\left(\sigma\left(A_{j}\right)\right)>0, j=1,2,3$. Then the fractional powers are well defined

$$
X^{\gamma}=D\left(A_{1}^{\gamma}\right)=D\left(A_{2}^{\gamma}\right)=D\left(A_{3}^{\gamma}\right), \quad 0<\gamma<1,
$$

with the graph norm

$$
\|x\|_{X^{\gamma}}=\left\|A_{j}^{\gamma} x\right\|_{X}, \quad \text { for } x \in X^{\gamma}, \quad j=1,2,3 .
$$

Then from Ref. 27, we have the following embeddings:

$$
\begin{aligned}
& X^{\gamma} \subset W^{1, p}(\Omega) \quad \text { for } \gamma>\frac{1}{2}, \\
& X^{\gamma} \subset C^{0, r}(\bar{\Omega}) \quad \text { for } \frac{r}{2}+\frac{n}{2 p}<\gamma<\frac{1}{2}+\frac{n}{2 p}, \quad 0<r<1,
\end{aligned}
$$

where $C^{0, r}(\bar{\Omega})$ is the space of all Hölder continuous functions with exponent $r$ in $\Omega$. Notice that for

$$
\gamma \in\left(\frac{1}{2}, \frac{1}{2}+\frac{1}{2 p}\right) \text { for } p>n
$$

(3.18) and 3.19) are satisfied.

Moreover, since $A_{1}, A_{2}$ and $A_{3}$ are sectorial operators, then each of $-A_{1},-A_{2}$ and $-A_{3}$ is the infinitesimal generator of an analytic semigroup $\left\{e^{-t A_{j}}\right\}_{t \geq 0}, j=$ $1,2,3$. Therefore, there exists a positive constant $C_{\gamma}$ such that the following inequality holds27:

$$
\left\|A_{j}^{\gamma} e^{-A_{j} t} w\right\|_{X} \leq C_{\gamma} t^{-\gamma} e^{-\xi_{j} t}\|w\|_{X}, \quad \text { for } w \in X,
$$

where $0<\xi_{j}<\operatorname{Re}\left(\sigma\left(A_{j}\right)\right), j=1,2,3$, and

$$
\left\|A_{j}^{\gamma} e^{-A_{j} t} w\right\|_{X} \leq k_{\gamma}\|w\|_{X \gamma}, \quad \text { for } w \in X^{\gamma}
$$

where $k_{\gamma}$ positive constant.

Theorem 3.1. Let $u_{1}^{\epsilon}(0, x), u_{2}^{\epsilon}(0, x), b^{\epsilon}(0, x) \in X^{\gamma}$ and $f^{\epsilon}(0, x), c^{\epsilon}(0, x) \in W^{1, p}$ $(\Omega)$. If assumptions (3.3) -3.13) and (3.20) are satisfied, then for any $T>0$ there exists a unique global-in-time solution $\left(u_{1}^{\epsilon}, u_{2}^{\epsilon}, f^{\epsilon}, c^{\epsilon}, b^{\epsilon}\right) \in C\left([0, T) ;\left[X^{\gamma}\right]^{2} \times\right.$ $\left.\left[W^{1, p}(\Omega)\right]^{2} \times X^{\gamma}\right)$ to (3.1)-(3.5), which remains bounded and the bounds are $\epsilon$-independent. Moreover, the solution satisfies

$$
\begin{aligned}
\left(u_{1}^{\epsilon}, u_{2}^{\epsilon}, b^{\epsilon}\right) & \in C^{1}\left((0, T) ;\left[X^{\gamma}\right]^{3}\right) \cap C\left((0, T) ;\left[W^{2, p}(\Omega)\right]^{3}\right), \\
f^{\epsilon}, c^{\epsilon} & \in C^{1}\left((0, T) ; W^{1, p}(\Omega)\right) .
\end{aligned}
$$

Proof. We will prove the existence of a local-in-time solution using the Banach contraction theorem. We first focus on the ODEs (3.1c) and (3.1d). We notice that relation (3.18) implies that $u_{1}^{\epsilon}, u_{2}^{\epsilon}, b^{\epsilon} \in W^{1, p}(\Omega)$, and since the functions $h_{2}$ : $\mathbb{R} \times \mathbb{R} \times \mathbb{R} \mapsto \mathbb{R}, h_{3}: \mathbb{R} \times \mathbb{R} \mapsto \mathbb{R}$ and $h_{4}: \mathbb{R} \mapsto \mathbb{R}$ are locally Lipschitz, we have by the property of superposition operator that the value of the functions $h_{2}, h_{3}$ and $h_{4}$ is also in $W^{1, p}(\Omega) ! \underline{58}$ The space $W^{1, p}(\Omega)$ for $p>n$ is an algebra with pointwise multiplication, and thus it follows that the functions $\left(u_{1}^{\epsilon}, u_{2}^{\epsilon}, f^{\epsilon}, b^{\epsilon}\right) \mapsto$ $P_{1}=h_{2}\left(u_{1}^{\epsilon}, u_{2}^{\epsilon}, b^{\epsilon}\right) f^{\epsilon}-\frac{\delta}{f_{m}} f^{\epsilon^{2}}$ and $\left(u_{1}^{\epsilon}, u_{2}^{\epsilon}, c^{\epsilon}, b^{\epsilon}\right) \mapsto P_{2}=h_{3}\left(u_{1}^{\epsilon}, u_{2}^{\epsilon}\right)+c^{\epsilon} h_{4}\left(b^{\epsilon}\right)$ are 
also $W^{1, p}$-valued. Since the right-hand side functions of Eqs. (3.1c) and (3.1d) are locally Lipschitz, it follows from assumption (3.10) and embeddings (3.18)-(3.19) that the mapping $P:\left(W^{1, p}(\Omega)\right)^{5} \mapsto\left(W^{1, p}(\Omega)\right)^{2}, P=\left(P_{1}, P_{2}\right)$, is a locally Lipschitz function.

For a fixed $T>0$ we note that functions:

$$
\begin{aligned}
& t \mapsto f_{0}+\int_{0}^{t} P_{1}\left(u_{1}^{\epsilon}(s), u_{2}^{\epsilon}(s), f^{\epsilon}(s), b^{\epsilon}(s)\right) \mathrm{d} s, \\
& t \mapsto c_{0}+\int_{0}^{t} P_{2}\left(u_{1}^{\epsilon}(s), u_{2}^{\epsilon}(s), c^{\epsilon}(s), b^{\epsilon}(s)\right) \mathrm{d} s,
\end{aligned}
$$

belong to the space $C\left([0, T] ; W^{1, p}(\Omega)\right)$.

Then, the system of the PDEs (3.1a), (3.1b) and (3.1e with (3.3) can be rewritten as:

$$
\begin{aligned}
z_{t} & =A z+H(z), & & \text { in } \Omega_{T}, \\
z(0, x) & =z_{0}(x), & & \text { in } \Omega,
\end{aligned}
$$

with

$$
z=\left(\begin{array}{l}
u_{1}^{\epsilon} \\
u_{2}^{\epsilon} \\
b^{\epsilon}
\end{array}\right), \quad A=\left(\begin{array}{ccc}
A_{1} & 0 & 0 \\
0 & A_{1} & 0 \\
0 & 0 & A_{3}
\end{array}\right)
$$

and

$$
H(z)=\left(\begin{array}{c}
-\nabla u_{1}^{\epsilon} \cdot F_{1}\left[u_{1}^{\epsilon}, u_{2}^{\epsilon}, f^{\epsilon}, c^{\epsilon}, b^{\epsilon}\right]-u_{1}^{\epsilon}\left(\nabla \cdot F_{1}\left[u_{1}^{\epsilon}, u_{2}^{\epsilon}, f^{\epsilon}, c^{\epsilon}, b^{\epsilon}\right]\right)+G_{1}(z) \\
-\nabla u_{2}^{\epsilon} \cdot F_{2}\left[u_{1}^{\epsilon}, u_{2}^{\epsilon}, f^{\epsilon}, c^{\epsilon}, b^{\epsilon}\right]-u_{2}^{\epsilon}\left(\nabla \cdot F_{2}\left[u_{1}^{\epsilon}, u_{2}^{\epsilon}, f^{\epsilon}, c^{\epsilon}, b^{\epsilon}\right]\right)+h_{1}(z) \\
h_{5}\left(u_{1}^{\epsilon}, u_{2}^{\epsilon}\right)
\end{array}\right)
$$

or, equivalently, we write that $A=A_{1} \times A_{2} \times A_{3}$ is sectorial in $X \times X \times X$, where $\left(A_{1} \times A_{2} \times A_{3}\right)\left(u_{1}^{\epsilon}, u_{2}^{\epsilon}, b^{\epsilon}\right)=\left(A_{1} u_{1}^{\epsilon}, A_{2} u_{2}^{\epsilon}, A_{3} b^{\epsilon}\right) \stackrel{27}{27}$ and the mapping $H:\left(X^{\gamma}\right)^{3} \mapsto$ $(X)^{3}, H=\left(H_{1}, H_{2}, H_{3}\right)$ is defined as the mapping of the right-hand side of Eqs. (3.1a), 3.1b and (3.1e). Using similar arguments as before for $P$, and assumption (3.9) we deduce that $H:\left(X^{\gamma}\right)^{3} \mapsto(X)^{3}$ is locally Lipschitz continuous.

Let us now denote $Y=X \times X \times W^{1, p}(\Omega) \times W^{1, p}(\Omega) \times X$. For a fixed $T>0$, we define the space

$$
Y_{T}^{\gamma}=C\left([0, T] ; Y^{\gamma}\right),
$$

where $Y^{\gamma}=X^{\gamma} \times X^{\gamma} \times W^{1, p}(\Omega) \times W^{1, p}(\Omega) \times X^{\gamma}$ equipped with the norm $\|y\|_{Y^{\gamma}}=$ $\max \left\{\left\|y_{1}\right\|_{X^{\gamma}},\left\|y_{2}\right\|_{X^{\gamma}},\left\|y_{3}\right\|_{W^{1, p}(\Omega)},\left\|y_{4}\right\|_{W^{1, p}(\Omega)},\left\|y_{5}\right\|_{X \gamma}\right\}$, for $y=\left(y_{1}, y_{2}, y_{3}, y_{4}, y_{5}\right) \in$ $Y^{\gamma}$.

We define the mapping $J: Y_{T}^{\gamma} \mapsto Y_{T}^{\gamma}$ with $J=\left(J_{1}, J_{2}, J_{3}, J_{4}, J_{5}\right)$ given by the following relation 


$$
J=\left\{\begin{array}{l}
J_{1}=e^{-A_{1} t} u_{1_{0}}+\int_{0}^{t} e^{-A_{1}(t-s)} H_{1}\left(u_{1}^{\epsilon}(s), u_{2}^{\epsilon}(s), f^{\epsilon}(s), c^{\epsilon}(s), b^{\epsilon}(s)\right) \mathrm{d} s \\
J_{2}=e^{-A_{2} t} u_{2_{0}}+\int_{0}^{t} e^{-A_{2}(t-s)} H_{2}\left(u_{1}^{\epsilon}(s), u_{2}^{\epsilon}(s), f^{\epsilon}(s), c^{\epsilon}(s), b^{\epsilon}(s)\right) \mathrm{d} s \\
J_{3}=f_{0}+\int_{0}^{t} P_{1}\left(u_{1}^{\epsilon}(s), u_{2}^{\epsilon}(s), f^{\epsilon}(s), b^{\epsilon}(s)\right) \mathrm{d} s \\
J_{4}=c_{0}+\int_{0}^{t} P_{2}\left(u_{1}^{\epsilon}(s), u_{2}^{\epsilon}(s), c^{\epsilon}(s), b^{\epsilon}(s)\right) \mathrm{d} s \\
J_{5}=e^{-A_{3} t} b_{0}+\int_{0}^{t} e^{-A_{3}(t-s)} H_{3}\left(u_{1}^{\epsilon}(s), u_{2}^{\epsilon}(s)\right) \mathrm{d} s .
\end{array}\right.
$$

Let $R>0$ be such that $\max \left\{\left\|u_{1_{0}}\right\|_{X^{\gamma}},\left\|u_{2_{0}}\right\|_{X^{\gamma}},\left\|b_{0}\right\|_{X^{\gamma}}\right\}<R /\left(2 k_{\gamma}\right)$, where $k_{\gamma}$ satisfies relation (3.22), and $\max \left\{\left\|f_{0}\right\|_{W^{1, p}(\Omega)},\left\|c_{0}\right\|_{W^{1, p}(\Omega)}\right\}<R / 2$ for $\left(u_{1_{0}}, u_{2_{0}}\right.$, $\left.f_{0}, c_{0}, b_{0}\right) \in Y^{\gamma}$. We define the ball

$$
B_{R}=\left\{y \in Y_{T}^{\gamma}:\|y\|_{Y_{T}^{\gamma}} \leq R\right\} \subset Y_{T}^{\gamma} .
$$

Thus, there exists $M_{R}>0$ such that $\sup _{y \in B_{R}}\left\|\left(H_{1}, H_{2}, P_{1}, P_{2}, H_{3}\right)(y)\right\|_{Y}<M_{R}$. We show that $J$ maps $B_{R}$ into itself and that $J$ is a strict contraction. By using relations (3.21)-(3.22) and (3.29) we obtain, for $T$ small enough,

$$
\begin{aligned}
\left\|J_{1}\left[u_{1}^{\epsilon}, u_{2}^{\epsilon}, f^{\epsilon}, c^{\epsilon}, b^{\epsilon}\right](t)\right\|_{X^{\gamma}} & \leq k_{\gamma}\left\|u_{1_{0}}\right\|_{X^{\gamma}}+M_{R} \int_{0}^{T} C_{\gamma}(t-s)^{-\gamma} e^{-\xi_{1}(t-s)} \mathrm{d} s \\
& \leq \frac{R}{2}+M_{R} \frac{C_{\gamma}}{1-\gamma} T^{1-\gamma}
\end{aligned}
$$

Similarly we obtain

$$
\left\|J_{2}\left[u_{1}^{\epsilon}, u_{2}^{\epsilon}, f^{\epsilon}, c^{\epsilon}, b^{\epsilon}\right](t)\right\|_{X^{\gamma}} \leq \frac{R}{2}+M_{R} \frac{C_{\gamma}}{1-\gamma} T^{1-\gamma}
$$

and

$$
\left\|J_{5}\left[u_{1}^{\epsilon}, u_{2}^{\epsilon}\right](t)\right\|_{X^{\gamma}} \leq \frac{R}{2}+M_{R} \frac{C_{\gamma}}{1-\gamma} T^{1-\gamma}
$$

Moreover, for $J_{3}$ and $J_{4}$ we have

$$
\left\|J_{l}\left[u_{1}^{\epsilon}, u_{2}^{\epsilon}, f^{\epsilon}, c^{\epsilon}, b^{\epsilon}\right](t)\right\|_{W^{1, p}(\Omega)} \leq \frac{R}{2}+M_{R} T, \quad l=3,4 .
$$

Hence, we can choose $T$ sufficiently small such that $\left\{M_{R} \frac{C_{\gamma}}{1-\gamma} T^{1-\gamma}, M_{R} T\right\}<\frac{R}{2}$, to assert that $J\left(B_{R}\right) \subset B_{R}$. We note also that $J\left[u_{1}^{\epsilon}, u_{2}^{\epsilon}, f^{\epsilon}, c^{\epsilon}, b^{\epsilon}\right]$ is continuous from $[0, T]$ to $E^{\gamma}$, as it can be easily proved using inequality (3.22). Thus $J$ maps $B_{R}$ into itself. 
If $y^{1}, y^{2} \in B_{R}$ then for $t \in[0, T]$ we have

$$
\begin{aligned}
\left\|J_{1}\left[y^{1}\right](t)-J_{1}\left[y^{2}\right](t)\right\|_{X^{\gamma}} & \leq \int_{0}^{t}\left\|A_{1}^{\gamma} e^{-\xi_{1}(t-s)}\right\|_{X}\left\|H_{1}\left(y^{1}(s)\right)-H_{1}\left(y^{2}(s)\right)\right\|_{X} \mathrm{~d} s \\
& \leq C_{\gamma} L_{R} \int_{0}^{T}(t-s)^{-\gamma} e^{\xi_{1}}(t-s)\left\|y^{1}-y^{2}\right\|_{Y_{T}^{\gamma}},
\end{aligned}
$$

where $L_{R}$ is the Lipschitz constant of $H$. Similar estimates can be obtained for the differences of the rest of the arguments. Therefore, it follows that

$$
\left\|J\left[y^{1}\right]-J\left[y^{2}\right]\right\|_{Y_{T}^{\gamma}} \leq \frac{1}{2}\left\|y^{1}-y^{2}\right\|_{Y_{T}^{\gamma}} \quad \text { for all } y^{1}, y^{2} \in B_{R} .
$$

Hence for $T$ small enough $J$ is a contraction mapping. Therefore, by Banach fixed point theorem, $J$ has a unique fixed point in $B_{R}$. Moreover, functions $f^{\epsilon}, c^{\epsilon}:[0, T] \mapsto C(\Omega)$ are locally Lipschitz, thus $f^{\epsilon}, c^{\epsilon} \in W^{1, \infty}\left([0, T] ; W^{1, p}(\Omega)\right)$ (see Theorem 4, Sec. 5.8.2 in Ref. 21). Therefore, it follows from Sec. 3.3 in Ref. 27 that there is a maximal time of existence $T_{\max }$ of regular solution $\left(u_{1}^{\epsilon}, u_{2}^{\epsilon}, b^{\epsilon}\right) \in$ $C\left(\left[0, T_{\max }\right) ;\left(X^{\gamma}\right)^{3}\right)$ such that for $t \in\left(0, T_{\max }\right)$ we have $\left(u_{1}^{\epsilon}, u_{2}^{\epsilon}, b^{\epsilon}\right) \in D(A)$. Then, from Ref. 27 (Sec. 3.3 and Theorem 3.5.2 in Sec. 3.5) we obtain that $\left(u_{1}^{\epsilon}, u_{2}^{\epsilon}, b^{\epsilon}\right) \in C^{\zeta}\left(\left(0, T_{\max }\right) ;\left(X^{\beta}\right)^{3}\right)$ for some $\zeta, \beta \in(0,1)$. Hence 3.1a), 3.1b and (3.1e) are satisfied in a pointwise manner on $\left(0, T_{\max }\right) \times \Omega$. It follows now from relations (3.8) and (3.19), and the regularity theory of parabolic systems, that $u_{1}^{\epsilon}, u_{2}^{\epsilon}$ and $b^{\epsilon}$ are classical solutions of (3.1a), (3.1b) and (3.1e), respectively.

Let us now prove the uniqueness of solution. Let $y^{1}=\left(u_{1}^{1}, u_{2}^{1}, f^{1}, c^{1}, b^{1}\right)$ and $y^{2}=$ $\left(u_{1}^{2}, u_{2}^{2}, f^{2}, c^{2}, b^{2}\right)$ be two solutions of system (3.1), with the same initial conditions. By linearity $y=y^{1}-y^{2}$ is a solution of (3.1) with zero initial conditions. Then, since all nonlinear terms are Lipschitz continuous and the components of the solution are $L^{\infty}$-bounded functions on bounded time intervals, we have

$$
\frac{\mathrm{d}}{\mathrm{d} t}\|y\|_{L^{2}(\Omega)}^{2} \leq k_{0}\|y\|_{L^{2}(\Omega)}^{2}, \quad \text { for } t \in\left[0, T_{\max }\right), \quad y(0)=0,
$$

for a constant $k_{0}$, and since $\|y(0)\|_{L^{2}(\Omega)}=0$, Gronwall's inequality implies that $\|y(t)\|_{L^{2}(\Omega)}=0$ for all $t \geq 0$, so $y=0$.

The equation for the ECM density given by 3.1c does not involve any spatial derivatives and $x$ behaves as a parameter. Thus, it is an ordinary differential equation in which the right-hand side is zero when $f^{\epsilon}(t, x)=0$ and for which local Lipschitz conditions hold. Therefore, from Picard-Lindelöf theorem we obtain a local unique solution for the initial value problem (3.1c), with $f^{\epsilon}(0, x)=0$. In the same way, we obtain a local unique solution for the initial value problem (3.1c), with $f^{\epsilon}(0, x) \geq 0$. Therefore, since $f^{\epsilon}(0, x) \geq 0$, from uniqueness of solutions we have $f^{\epsilon}(t, x) \geq 0$ for all $t>0, x \in \Omega$. Then from maximum principle arguments it follows from system (3.27) that $u_{1}^{\epsilon}, u_{2}^{\epsilon}, b^{\epsilon} \geq 0$ on $\left[0, T_{\max }\right) \times \Omega$.

Finally, the equation for the integrin density given by (3.1d) can be treated in a similar manner as Eq. (3.1C) for the ECM density. Again we have an ordinary differential equation in which the right-hand side is greater than or equal to zero when 
$c^{\epsilon}(t, x)=0$, and for which local Lipschitz conditions hold. Therefore, $c^{\epsilon}(t, x) \geq 0$ on $\left[0, T_{\max }\right) \times \Omega$.

We proceed now with the proof of global-in-time solution. Let us first integrate Eq. (3.1a) on $\Omega$. Then, from the boundary conditions (3.4) 3.5 and relation (3.11) we obtain

$$
\frac{\mathrm{d}}{\mathrm{d} t} \int_{\Omega} u_{1}^{\epsilon}(t, x) \mathrm{d} x \leq B_{1}|\Omega|-\left(M+D_{1}\right) \int_{\Omega} u_{1}^{\epsilon}(t, x) \mathrm{d} x .
$$

Thus, Gronwall's inequality yields the estimate

$$
\sup _{t \in\left[0, T_{\max }\right)}\left\|u_{1}^{\epsilon}(t, \cdot)\right\|_{L^{1}(\Omega)} \leq \max \left\{\frac{B_{1}|\Omega|}{M+D_{1}},\left\|u_{1_{0}}\right\|_{L^{1}(\Omega)}\right\}:=M_{u_{1}} .
$$

Similarly, we have

$$
\sup _{t \in\left[0, T_{\max }\right)}\left\|u_{2}^{\epsilon}(t, \cdot)\right\|_{L^{1}(\Omega)} \leq \max \left\{\frac{M M_{u_{1}}+B_{2}|\Omega|}{D_{2}},\left\|u_{2_{0}}\right\|_{L^{1}(\Omega)}\right\}:=M_{u_{2}}
$$

and

$$
\sup _{t \in\left[0, T_{\max }\right)}\left\|b^{\epsilon}(t, \cdot)\right\|_{L^{1}(\Omega)} \leq \max \left\{\frac{\mu_{1} M_{u_{1}}+\mu_{2} M_{u_{2}}}{q_{b}},\left\|b_{0}\right\|_{L^{1}(\Omega)}\right\}:=M_{b},
$$

hence from relations 3.12 -3.13 and Gronwall's inequality again we have

$$
\sup _{t \in\left[0, T_{\max }\right)}\left\|f^{\epsilon}(t, \cdot)\right\|_{L^{1}(\Omega)} \leq \max \left\{\frac{\Lambda_{1} M_{b}}{\Lambda_{2}},\left\|f_{0}\right\|_{L^{1}(\Omega)}\right\}:=M_{f}
$$

and

$$
\sup _{t \in\left[0, T_{\max }\right)}\left\|c^{\epsilon}(t, \cdot)\right\|_{L^{1}(\Omega)} \leq \max \left\{\frac{p_{1} M_{u_{1}}+p_{2} M_{u_{2}}+\Lambda_{3} M_{b}}{\Lambda_{4}},\left\|c_{0}\right\|_{L^{1}(\Omega)}\right\}:=M_{c} .
$$

Thus, from relations (3.6) -3.9 we have for all $t \in\left[0, T_{\max }\right)$ :

$$
\begin{aligned}
& \left\|\left(\sum_{j=1}^{n} \partial_{x_{j}} F_{i}\left[u_{1}^{\epsilon}, u_{2}^{\epsilon}, f^{\epsilon}, c^{\epsilon}, b^{\epsilon}\right]\right)+F_{i}\left[u_{1}^{\epsilon}, u_{2}^{\epsilon}, f^{\epsilon}, c^{\epsilon}, b^{\epsilon}\right]\right\|_{L^{\infty}(\Omega)} \leq L_{N}\left(\left\|u_{1}^{\epsilon}(t)\right\|_{L^{1}(\Omega)}\right. \\
& \left.\quad+\left\|u_{2}^{\epsilon}(t)\right\|_{L^{1}(\Omega)}+\left\|f^{\epsilon}(t)\right\|_{L^{1}(\Omega)}\right) \leq L_{N}\left(M_{u_{1}}+M_{u_{2}}+M_{f}\right), \quad i=1,2 . \quad
\end{aligned}
$$

From system (3.27), we rewrite the elliptic operators in the form:

$$
\begin{aligned}
& -D_{u} \Delta u_{1}^{\epsilon}+\sum_{j=1}^{n} b_{1}^{j} \partial_{x_{j}} u_{1}^{\epsilon}+d_{1} u_{1}^{\epsilon}, \\
& -\epsilon \Delta u_{2}^{\epsilon}+\sum_{j=1}^{n} b_{2}^{j} \partial_{x_{j}} u_{2}^{\epsilon}+d_{2} u_{2}^{\epsilon}, \\
& -D_{b} \Delta b^{\epsilon}+q_{b} b^{\epsilon},
\end{aligned}
$$

where we denote by $b_{i}^{j}=F_{i_{j}}\left[u_{1}^{\epsilon}, u_{2}^{\epsilon}, f^{\epsilon}, c^{\epsilon}, b^{\epsilon}\right]$ and by $d_{i}=\sum_{j=1}^{n} \partial_{x_{j}} F_{i}\left[u_{1}^{\epsilon}, u_{2}^{\epsilon}, f^{\epsilon}\right.$, $\left.c^{\epsilon}, b^{\epsilon}\right], i=1,2, j=1, \ldots, n$. From relation (3.43) it follows that $b_{i}^{j}$ and $d_{i}, i=1,2$, are 
bounded on $(0, \infty) \times \Omega$. Hence, from the fact that the reaction terms are dissipative (see Ref. 13), it follows by Möser-Alikakos method (see Sec. 9.3 in Ref. 13) that the uniform in time $L^{1}(\Omega)$ estimate implies the uniform in time $L^{\infty}(\Omega)$ estimate for the solution $\left(u_{1}^{\epsilon}, u_{2}^{\epsilon}, b^{\epsilon}\right)$ of (3.27). Therefore, there is a constant $M_{\infty}$ independent of $\epsilon$ (see Remark 3.1), such that

$$
\sup _{t \in\left[0, T_{\max }\right)}\left(\left\|u_{1}^{\epsilon}(t)\right\|_{L^{\infty}(\Omega)}+\left\|u_{2}^{\epsilon}(t)\right\|_{L^{\infty}(\Omega)}+\left\|b^{\epsilon}(t)\right\|_{L^{\infty}(\Omega)}\right)<M_{\infty} .
$$

Moreover, from 3.12 -3.13) and 3.44), and the comparison theorem it follows that

$$
\sup _{t \in\left[0, T_{\max }\right)}\left\|f^{\epsilon}(t)\right\|_{L^{\infty}(\Omega)}<\max \left\{\left\|f_{0}\right\|_{L^{\infty}(\Omega)}, \frac{\Lambda_{1} M_{\infty}}{\Lambda_{2}}\right\}
$$

and

$$
\sup _{t \in\left[0, T_{\max }\right)}\left\|c^{\epsilon}(t)\right\|_{L^{\infty}(\Omega)}<\max \left\{\left\|c_{0}\right\|_{L^{\infty}(\Omega)},\left(p_{1}+p_{2}\right) M_{\infty}, \frac{\Lambda_{3} M_{\infty}}{\Lambda_{4}}\right\} .
$$

Relations 3.44 $-(3.46)$ can be used to show that

$$
\left\|H_{i}\left(u_{1}^{\epsilon}(s), u_{2}^{\epsilon}(s), f^{\epsilon}(s), c^{\epsilon}(s), b^{\epsilon}(s)\right)\right\|_{X} \leq M_{\gamma_{i}}\left(1+\left\|u_{i}^{\epsilon}(t)\right\|_{X^{\gamma}}\right) \quad \text { for } t \in\left[0, T_{\max }\right)
$$

and

$$
\left\|H_{3}\left(u_{1}^{\epsilon}(s), u_{2}^{\epsilon}(s)\right)\right\|_{X} \leq M_{\gamma_{3}} \text { for } t \in\left[0, T_{\max }\right),
$$

where $M_{\gamma_{i}}, M_{\gamma_{3}}, i=1,2$, are constants depending on $M_{\infty}$.

We show now the global existence of solution by contradiction. Let us suppose that for $T_{\max }<\infty$ we have

$$
\begin{aligned}
& \sup _{t \in\left[0, T_{\max }\right)}\left(\left\|u_{1}^{\epsilon}(t)\right\|_{X^{\gamma}}+\left\|u_{2}^{\epsilon}(t)\right\|_{X^{\gamma}}+\left\|f^{\epsilon}(t)\right\|_{W^{1, p}(\Omega)}+\left\|c^{\epsilon}(t)\right\|_{W^{1, p}(\Omega)}\right. \\
& \left.\quad+\left\|b^{\epsilon}(t)\right\|_{X^{\gamma}}\right) \rightarrow \infty \quad \text { as } t \rightarrow T_{\max } .
\end{aligned}
$$

From relation (3.47) and Volterra-type integral inequality 13 it follows, as in Corollary 3.3.5 in Ref. 27, that

$$
\sup _{t \in\left[0, T_{\max }\right)}\left\|u_{i}^{\epsilon}(t)\right\|_{X^{\gamma}} \leq\left(k_{\gamma}\left\|u_{i_{0}}^{\epsilon}\right\|_{X^{\gamma}}+C_{\gamma} M_{\gamma_{i}} \int_{0}^{T_{\max }} \frac{e^{-\xi_{i}(t-s)}}{(t-s)^{\gamma}}\right) C_{T_{\max }}
$$

where $C_{T_{\max }}:=C_{C_{\gamma} M_{\gamma_{i}}, \gamma, T_{\max }}, i=1,2$, is a continuous function increasing with respect to $T_{\max }$, while for the function $b^{\epsilon}$ we have from relation (3.48) that

$$
\sup _{t \in\left[0, T_{\max }\right)}\left\|b^{\epsilon}(t)\right\|_{X^{\gamma}} \leq\left(k_{\gamma}\left\|b_{0}^{\epsilon}\right\|_{X^{\gamma}}+C_{\gamma} M_{\gamma_{3}} \int_{0}^{T_{\max }} \frac{e^{-\xi_{3}(t-s)}}{(t-s)^{\gamma}}\right) .
$$

Note that $k_{\gamma}$ and $C_{\gamma}$ are $\epsilon$-independent constants since $0<\epsilon \leq 1$ (see Theorem 1.3.4 in Ref. 27). We conclude that

$$
\sup _{t \in\left[0, T_{\max }\right)}\left(\left\|u_{1}^{\epsilon}(t)\right\|_{X^{\gamma}}+\left\|u_{2}^{\epsilon}(t)\right\|_{X^{\gamma}}+\left\|b^{\epsilon}(t)\right\|_{X^{\gamma}}\right)<\infty .
$$


Whence, by 3.18 it follows now that

$$
\sup _{t \in\left[0, T_{\max }\right)}\left(\left\|u_{1}^{\epsilon}(t)\right\|_{W^{1, p}(\Omega)}+\left\|u_{2}^{\epsilon}(t)\right\|_{W^{1, p}(\Omega)}+\left\|b^{\epsilon}(t)\right\|_{W^{1, p}(\Omega)}\right)<\infty .
$$

By Eq. 3.1c and direct calculations, we obtain

$$
\nabla f_{t}^{\epsilon}=\left(-\alpha \nabla u_{1}^{\epsilon}-\beta \nabla u_{2}^{\epsilon}+\theta_{\beta} \nabla b^{\epsilon}\right) f^{\epsilon}+h_{6} \nabla f^{\epsilon},
$$

where

$$
h_{6}:=h_{2}-\frac{2 \delta}{f_{m}} f^{\epsilon}=-\alpha u_{1}^{\epsilon}-\beta u_{2}^{\epsilon}+\theta_{\beta} b^{\epsilon}+\delta-\frac{2 \delta}{f_{m}} f^{\epsilon} \leq \theta_{\beta} b^{\epsilon}+\delta .
$$

For notational convenience, in what follows we denote various non-negative constants, which are independent of $T$ or $t$, by $c_{j}, j=1,2, \ldots, 8$.

Multiplying (3.52) by $p \nabla f^{\epsilon}\left|\nabla f^{\epsilon}\right|^{p-2}$, using (3.53), Young's inequality, and the estimates 3.44, 3.45 and (3.51), and integrating over $\Omega$, we deduce that

$$
\begin{aligned}
\frac{d}{d t}\left\|\nabla f^{\epsilon}\right\|_{L^{p}(\Omega)}^{p} \leq & p \int_{\Omega}\left(-\alpha \nabla u_{1}^{\epsilon}-\beta \nabla u_{2}^{\epsilon}+\theta_{\beta} \nabla b^{\epsilon}\right) f^{\epsilon} \nabla f^{\epsilon}\left|\nabla f^{\epsilon}\right|^{p-2} \mathrm{~d} x \\
& +p \theta_{\beta} \int_{\Omega} b^{\epsilon}\left|\nabla f^{\epsilon}\right|^{p} \mathrm{~d} x+p \delta\left\|\nabla f^{\epsilon}\right\|_{L^{p}(\Omega)}^{p} \\
\leq & p \max \left\{\alpha, \beta, \theta_{\beta}\right\}\left\|f^{\epsilon}\right\|_{L^{\infty}(\Omega)}\left(\int_{\Omega}\left|\nabla u_{1}^{\epsilon} \| \nabla f^{\epsilon}\right|^{p-1} \mathrm{~d} x\right. \\
& +\int_{\Omega}\left|\nabla u_{2}^{\epsilon}\left\|\left.\nabla f^{\epsilon}\right|^{p-1} \mathrm{~d} x+\int_{\Omega}\left|\nabla b^{\epsilon} \| \nabla f^{\epsilon}\right|^{p-1} \mathrm{~d} x\right)\right. \\
& +p\left(\theta_{\beta}\left\|b^{\epsilon}\right\|_{L^{\infty}(\Omega)}+\delta\right)\left\|\nabla f^{\epsilon}\right\|_{L^{p}(\Omega)}^{p} \\
\leq & c_{1}\left(\left\|\nabla u_{1}^{\epsilon}\right\|_{L^{p}(\Omega)}^{p}+\left\|\nabla u_{2}^{\epsilon}\right\|_{L^{p}(\Omega)}^{p}+\left\|\nabla b^{\epsilon}\right\|_{L^{p}(\Omega)}^{p}\right)+c_{2}\left\|\nabla f^{\epsilon}\right\|_{L^{p}(\Omega)}^{p} .
\end{aligned}
$$

By Gronwall's inequality and previous estimates, we have

$$
\sup _{t \in\left[0, T_{\max }\right)}\left\|f^{\epsilon}(t)\right\|_{W^{1, p}(\Omega)} \leq c_{3} e^{c_{4} t}, \quad \text { for } t \geq 0 .
$$

Similarly, by Eq. (3.1d and direct calculations, we obtain

$$
\nabla c_{t}^{\epsilon}=p_{1} \nabla u_{1}^{\epsilon}+p_{2} \nabla u_{2}^{\epsilon}+p_{3} \nabla b^{\epsilon} c^{\epsilon}+p_{3} b^{\epsilon} \nabla c^{\epsilon}-q \nabla c^{\epsilon} .
$$

Multiplying now (3.55) by $p \nabla c^{\epsilon}\left|\nabla c^{\epsilon}\right|^{p-2}$, using the estimates (3.44), (3.46) and (3.51), as well as Young's inequality, and integrating over $\Omega$, we obtain

$$
\begin{aligned}
\frac{d}{d t}\left\|\nabla c^{\epsilon}\right\|_{L^{p}(\Omega)}^{p} \leq & p p_{1} \int_{\Omega} \nabla u_{1}^{\epsilon} \nabla c^{\epsilon}\left|\nabla c^{\epsilon}\right|^{p-2} \mathrm{~d} x+p p_{2} \int_{\Omega} \nabla u_{2}^{\epsilon} \nabla c^{\epsilon}\left|\nabla c^{\epsilon}\right|^{p-2} \mathrm{~d} x \\
& +p p_{3} \int_{\Omega} \nabla b^{\epsilon} c^{\epsilon} \nabla c^{\epsilon}\left|\nabla c^{\epsilon}\right|^{p-2} \mathrm{~d} x+p p_{3} \int_{\Omega} b^{\epsilon}\left|\nabla c^{\epsilon}\right|^{p} \mathrm{~d} x-p q \int_{\Omega}\left|\nabla c^{\epsilon}\right|^{p} \mathrm{~d} x \\
\leq & p \max \left\{p_{1}, p_{2}, p_{3}\left\|c^{\epsilon}\right\|_{L^{\infty}(\Omega)}\right\}\left(\int_{\Omega}\left|\nabla u_{1}^{\epsilon} \| \nabla c^{\epsilon}\right|^{p-1} \mathrm{~d} x\right.
\end{aligned}
$$




$$
\begin{aligned}
& \left.+\int_{\Omega}\left|\nabla u_{2}^{\epsilon}\right|\left|\nabla c^{\epsilon}\right|^{p-1} \mathrm{~d} x+\int_{\Omega}\left|\nabla b^{\epsilon}\right|\left|\nabla c^{\epsilon}\right|^{p-1} \mathrm{~d} x\right) \\
& +p\left(p_{3}\left\|b^{\epsilon}\right\|_{L^{\infty}(\Omega)}-q\right)\left\|\nabla c^{\epsilon}\right\|_{L^{p}(\Omega)}^{p} \\
& \leq c_{5}\left(\left\|\nabla u_{1}^{\epsilon}\right\|_{L^{p}(\Omega)}^{p}+\left\|\nabla u_{2}^{\epsilon}\right\|_{L^{p}(\Omega)}^{p}+\left\|\nabla b^{\epsilon}\right\|_{L^{p}(\Omega)}^{p}\right)+c_{6}\left\|\nabla c^{\epsilon}\right\|_{L^{p}(\Omega)}^{p} .
\end{aligned}
$$

This, together with Gronwall's inequality and previous estimates, yields

$$
\sup _{t \in\left[0, T_{\max }\right)}\left\|c^{\epsilon}(t)\right\|_{W^{1, p}(\Omega)} \leq c_{7} e^{c_{8} t}, \quad \text { for } t \geq 0 .
$$

Bounds (3.50), (3.54) and (3.56) contradict (3.49), therefore the solution exists globally.

Remark 3.1. It is easy to see how the proof of Möser-Alikakos method in Ref. 13 can be used in our case to show the $\epsilon$-independent $L^{\infty}$-estimates given in relation (3.44). Following along the same lines with the proof of Lemma 9.3.1 in Ref. 13, we obtain a constant $c^{\prime}$ (as described in relation (9.3.11) of the proof in Ref. 13) such that

$$
c^{\prime}:=\max \left\{\text { const. } a_{0}, 1, D|\Omega|, K^{2}\right\}
$$

where $a_{0}$ will be each of the diffusion coefficients $D_{u}, \epsilon$ and $D_{b}$. Using the fact that $0<D_{u}, \epsilon, D_{b} \leq 1$ it follows that $c^{\prime}$ is $\epsilon$-independent.

By this result, it follows that the rest of the estimates given by relations (3.45)(3.48), 3.50-3.51), 3.54 and (3.56) are $\epsilon$-independent.

\subsection{Vanishing viscosity limit}

Now we are ready to take the vanishing viscosity limit $\epsilon \rightarrow 0$ and prove the existence of solution for system (2.10). First we introduce the notion of a weak solution to problem (2.10) $-(2.11)$ with (2.12) $-(2.13)$.

Definition 3.1. A function $\left(u_{1}, u_{2}, f, c, b\right) \in L^{\infty}\left(\bar{\Omega}_{T}\right) \cap L^{\infty}\left(0, T ;\left[L^{1}(\Omega)\right]^{5}\right)$ is called a weak solution of the problem (2.10)-2.11) with (2.12)-(2.13) if:

(i) For all $\phi \in C_{0}^{\infty}\left(\Omega_{T}\right)$ we have

$$
\begin{aligned}
& -\int_{\bar{\Omega}_{T}} u_{1} \phi_{t}-D_{u} \nabla u_{1} \cdot \nabla \phi+u_{1} F_{1}[\underline{u}, f, c, b] \cdot \nabla \phi \\
& \quad=\int_{\bar{\Omega}_{T}}\left[-M u_{1}+G_{1}(\underline{u}, b)\right] \phi \\
& -\int_{\bar{\Omega}_{T}} u_{2} \phi_{t}+u_{2} F_{2}[\underline{u}, f, c, b] \cdot \nabla \phi=\int_{\bar{\Omega}_{T}}\left[M u_{1}+G_{2}(\underline{u}, b)\right] \phi,
\end{aligned}
$$




$$
\begin{aligned}
& -\int_{\bar{\Omega}_{T}} f \phi_{t}=\int_{\bar{\Omega}_{T}}\left[-\alpha u_{1} f-\beta u_{2} f+\theta_{\beta} b f+\delta f\left(1-\frac{f}{f_{m}}\right)\right] \phi, \\
& -\int_{\bar{\Omega}_{T}} c \phi_{t}=\int_{\bar{\Omega}_{T}}\left[p_{1} u_{1}+p_{2} u_{2}+p_{3} b c-q c\right] \phi, \\
& -\int_{\bar{\Omega}_{T}} b \phi_{t}-D_{b} \nabla b \cdot \nabla \phi=\int_{\bar{\Omega}_{T}}\left[\mu_{1} u_{1}+\mu_{2} u_{2}-q_{b} b\right] \phi .
\end{aligned}
$$

(ii) The functions $u_{1}, u_{2}, f, c$ and $b$ satisfy the initial conditions $u_{1_{0}}(x), u_{2_{0}}(x)$, $f_{0}(x), c_{0}(x)$ and $b_{0}(x)$, respectively, given by (2.11), in the weak sense, i.e. there exists a set $E \subset[0, T]$ of Lebesgue measure zero such that $u_{1}\left(t_{0}, \cdot\right), u_{2}\left(t_{0}, \cdot\right), f\left(t_{0}, \cdot\right), c\left(t_{0}, \cdot\right)$ and $b\left(t_{0}, \cdot\right)$ are defined almost everywhere in $\Omega$ for $t_{0} \in[0, T] \backslash E$ and satisfy:

$$
\begin{aligned}
\lim _{t_{0} \rightarrow 0, t_{0} \in[0, T] \backslash E} \int_{\Omega}\left|u_{i}\left(t_{0}, x\right)-u_{i_{0}}(x)\right| \mathrm{d} x & =0, \quad i=1,2, \\
\lim _{t_{0} \rightarrow 0, t_{0} \in[0, T] \backslash E} & \int_{\Omega}\left|f\left(t_{0}, x\right)-f_{0}(x)\right| \mathrm{d} x=0, \\
\lim _{t_{0} \rightarrow 0, t_{0} \in[0, T] \backslash E} \int_{\Omega}\left|c\left(t_{0}, x\right)-c_{0}(x)\right| \mathrm{d} x & =0, \\
\lim _{t_{0} \rightarrow 0, t_{0} \in[0, T] \backslash E} \int_{\Omega}\left|b\left(t_{0}, x\right)-b_{0}(x)\right| \mathrm{d} x & =0 .
\end{aligned}
$$

We show now the $L^{1}$-estimates with respect to time, which will be used in the proof of existence of solution to model (2.10).

Theorem 3.2. Let the assumptions of Theorem 3.24 hold. Then for each $\rho>$ 0 there exist nondecreasing functions $\omega_{\rho}^{u_{i}}, \omega_{\rho}^{f}, \omega_{\rho}^{c}, \omega_{\rho}^{b} \in C([0, \infty))$ with $\omega_{\rho}^{u_{i}}(0)=$ $\omega_{\rho}^{f}(0)=\omega_{\rho}^{c}(0)=\omega_{\rho}^{b}(0)=0, i=1,2$, such that for any $\epsilon \in(0,1]$ and for any $t$, $t+\Delta t \in[0, T], \Delta t \geq 0$ we have for a ball $B_{\rho}=\{|x| \leq \rho\}$ that:

$$
\begin{aligned}
& \int_{B_{\rho}}\left|u_{i}^{\epsilon}(t+\Delta t, x)-u_{i}^{\epsilon}(t, x)\right| \mathrm{d} x \leq \omega_{\rho}^{u_{i}}(\Delta t), \quad i=1,2 \\
& \int_{B_{\rho}}\left|f^{\epsilon}(t+\Delta t, x)-f^{\epsilon}(t, x)\right| \mathrm{d} x \leq \omega_{\rho}^{f}(\Delta t) \\
& \int_{B_{\rho}}\left|c^{\epsilon}(t+\Delta t, x)-c^{\epsilon}(t, x)\right| \mathrm{d} x \leq \omega_{\rho}^{c}(\Delta t) \\
& \int_{B_{\rho}}\left|b^{\epsilon}(t+\Delta t, x)-b^{\epsilon}(t, x)\right| \mathrm{d} x \leq \omega_{\rho}^{b}(\Delta t)
\end{aligned}
$$


Proof. Let us consider a function $g \in C_{0}^{2}(\Omega)$ with $\operatorname{supp}(g) \subset B_{\rho}$. Then from the estimates obtained by Theorem 3.24 we have

$$
\begin{aligned}
\left|\int_{\Omega}\left(u_{2}^{\epsilon}(t+\Delta t, x)-u_{2}^{\epsilon}(t, x)\right) g(x) \mathrm{d} x\right| \\
=\left|\int_{\Omega} g(x) \int_{t}^{t+\Delta t} u_{2_{t}}^{\epsilon}(s, x) \mathrm{d} s \mathrm{~d} x\right| \leq\left|\int_{t}^{t+\Delta t} \int_{\Omega} \epsilon u_{2}^{\epsilon}(s, x) \Delta g(x) \mathrm{d} x \mathrm{~d} s\right| \\
\quad+\left|\int_{t}^{t+\Delta t} \int_{\Omega} u_{2}^{\epsilon}(s, x) F_{2}\left[u_{1}^{\epsilon}, u_{2}^{\epsilon}, f^{\epsilon}, c^{\epsilon}, b^{\epsilon}\right] \nabla g(x) \mathrm{d} x \mathrm{~d} s\right| \\
\quad+\left|\int_{t}^{t+\Delta t} \int_{\Omega}\left[M u_{1}^{\epsilon}(s, x)+G_{2}\left(u_{1}^{\epsilon}, u_{2}^{\epsilon}, b^{\epsilon}\right)\right] g(x) \mathrm{d} x \mathrm{~d} s\right| \\
\leq C\left(\rho, M_{\infty}\right) \Delta t\|g\|_{C^{2}(\Omega)} .
\end{aligned}
$$

Similarly we can obtain estimates for $u_{1}, f, c$ and $b$. Then, as in Ref. 29, the $L^{1}$-estimates of $u_{1}^{\epsilon}, u_{2}^{\epsilon}, f^{\epsilon}, c^{\epsilon}$ and $b^{\epsilon}$ with respect to time $t$ follow.

Theorem 3.3. Let $u_{i}(0, x), b(0, x) \in X^{\gamma}, i=1,2$, and $f(0, x), c(0, x) \in W^{1, p}(\Omega)$. If assumptions of Theorem 3.24 are satisfied, then there exists a weak solution $\left(u_{1}, u_{2}, f, c, b\right)$ of model (2.10) with (2.11) -(2.13), such that for all $T>0$ the weak solution satisfies for almost all $(t, x) \in \bar{\Omega}_{T}$

$$
0 \leq u_{1}(t, x), u_{2}(t, x), f(t, x), c(t, x), b(t, x) \leq C\left(M_{\infty}\right) .
$$

Proof. By Theorem 3.24 we have that for all $0<\epsilon \leq 1$ and $T>0$ there exists a classical solution $\left(u_{1}^{\epsilon}, u_{2}^{\epsilon}, f^{\epsilon}, c^{\epsilon}, b^{\epsilon}\right)$ of problem 3.1 3.3 , which is uniformly bounded in $L^{\infty}\left(\bar{\Omega}_{T}\right)$. From estimates 3.51), 3.54 and 3.56) it follows that

$$
\begin{gathered}
\sup _{t \in[0, T)}\left(\left\|u_{1}^{\epsilon}(t)\right\|_{W^{1,1}(\Omega)}+\left\|u_{2}^{\epsilon}(t)\right\|_{W^{1,1}(\Omega)}+\left\|f^{\epsilon}(t)\right\|_{W^{1,1}(\Omega)}\right. \\
\left.+\left\|c^{\epsilon}(t)\right\|_{W^{1,1}(\Omega)}+\left\|b^{\epsilon}(t)\right\|_{W^{1,1}(\Omega)}\right)<C .
\end{gathered}
$$

We consider for $m \in \mathbb{N}$ a sequence $\epsilon_{m}$ with $\epsilon_{m} \rightarrow 0$ for $m \rightarrow \infty$. Then by estimate (3.72) and $L^{1}$-estimates with respect to time, obtained from Theorem 3.2 , it follows by Fréchet-Kolmogorov theorem that the sequences $\left\{u_{1}^{\epsilon_{m}}, u_{2}^{\epsilon_{m}}, f^{\epsilon_{m}}, c^{\epsilon_{m}}, b^{\epsilon_{m}}\right\}$ are precompact in $L_{\text {loc }}^{1}\left(\bar{\Omega}_{T}\right)$. Using a standard diagonal extraction argument we obtain subsequences, denoted also as $\left\{u_{1}^{\epsilon_{m}}, u_{2}^{\epsilon_{m}}, f^{\epsilon_{m}}, c^{\epsilon_{m}}, b^{\epsilon_{m}}\right\}$, and functions $u_{1}, u_{2}, f, c$, $b \in L_{\mathrm{loc}}^{1}\left(\bar{\Omega}_{T}\right)$ with $u_{i}^{\epsilon_{m}} \rightarrow u_{i}, i=1,2, f^{\epsilon_{m}} \rightarrow f, c^{\epsilon_{m}} \rightarrow c$ and $b^{\epsilon_{m}} \rightarrow b$ in $L_{\text {loc }}^{1}\left(\bar{\Omega}_{T}\right)$. This implies that the convergence is even pointwise a.e. for a suitable subsequence. From uniform $L^{1}$-bounds of $\left\{u_{1}^{\epsilon_{m}}(t, \cdot), u_{2}^{\epsilon_{m}}(t, \cdot), f^{\epsilon_{m}}(t, \cdot), c^{\epsilon_{m}}(t, \cdot), b^{\epsilon_{m}}(t, \cdot)\right\}$ we have that $u_{1}(t, \cdot), u_{2}(t, \cdot), f(t, \cdot), c(t, \cdot), b(t, \cdot) \in L^{1}(\Omega)$. Multiplying now Eqs. (2.10a)(2.10e) with a test function $\phi \in C_{0}^{\infty}\left(\Omega_{T}\right)$, taking the integral and integrating by 
parts over $\bar{\Omega}_{T}$ yields

$$
\begin{aligned}
& -\int_{\bar{\Omega}_{T}} u_{1}^{\epsilon_{m}} \phi_{t}+D_{u} u_{1}^{\epsilon_{m}} \cdot \Delta \phi+u_{1}^{\epsilon_{m}} F_{1}\left[\underline{u}^{\epsilon_{m}}, f^{\epsilon_{m}}, c^{\epsilon_{m}}, b^{\epsilon_{m}}\right] \cdot \nabla \phi \\
& =\int_{\bar{\Omega}_{T}}\left[-M u_{1}^{\epsilon_{m}}+G_{1}\left(\underline{u}^{\epsilon_{m}}, b^{\epsilon_{m}}\right)\right] \phi, \\
& -\int_{\bar{\Omega}_{T}} u_{2}^{\epsilon_{m}} \phi_{t}+u_{2}^{\epsilon_{m}} F_{2}\left[\underline{u}^{\epsilon_{m}}, f^{\epsilon_{m}}, c^{\epsilon_{m}}, b^{\epsilon_{m}}\right] \cdot \nabla \phi \\
& =\int_{\bar{\Omega}_{T}}\left[M u_{1}^{\epsilon_{m}}+G_{2}\left(\underline{u}^{\epsilon_{m}}, b^{\epsilon_{m}}\right)\right] \phi+\epsilon \int_{\bar{\Omega}_{T}} u_{2}^{\epsilon_{m}} \Delta \phi, \\
& -\int_{\bar{\Omega}_{T}} f^{\epsilon_{m}} \phi_{t}=\int_{\bar{\Omega}_{T}}\left[-\alpha u_{1}^{\epsilon_{m}} f^{\epsilon_{m}}-\beta u_{2}^{\epsilon_{m}} f^{\epsilon_{m}}\right. \\
& \left.+\theta_{\beta} b^{\epsilon_{m}} f^{\epsilon_{m}}+\delta f^{\epsilon_{m}}\left(1-f^{\epsilon_{m}} / f_{m}\right)\right] \phi, \\
& -\int_{\bar{\Omega}_{T}} c^{\epsilon_{m}} \phi_{t}=\int_{\bar{\Omega}_{T}}\left[p_{1} u_{1}^{\epsilon_{m}}+p_{2} u_{2}^{\epsilon_{m}}+p_{3} b^{\epsilon_{m}} c^{\epsilon_{m}}-q c^{\epsilon_{m}}\right] \phi, \\
& -\int_{\bar{\Omega}_{T}} b^{\epsilon_{m}} \phi_{t}+D_{b} b^{\epsilon_{m}} \cdot \Delta \phi=\int_{\bar{\Omega}_{T}}\left[\mu_{1} u_{1}^{\epsilon_{m}}+\mu_{2} u_{2}^{\epsilon_{m}}-q_{b} b^{\epsilon_{m}}\right] \phi .
\end{aligned}
$$

The last term in (3.74) vanishes in the limit due to the uniform $L^{\infty}$-bound on $\left\{u_{2}^{\epsilon_{m}}\right\}$ and from the pointwise convergence the Lebesgue's dominated convergence theorem ensures that the limit $\left(u_{1}, u_{2}, f, c, b\right)$ satisfies (3.58)-3.62). Moreover, $\left(u_{1}, u_{2}, f, c, b\right) \in L^{\infty}\left(\bar{\Omega}_{T}\right)$, and by relations (3.44)-(3.46) and Remark 3.1]we obtain the bounds (3.71).

It remains to show the initial conditions (3.63)-3.66). Let us first define the set $E \subset[0, T]$ such that for all $t_{0} \in[0, T] \backslash E$ we have for almost all $x \in \Omega$ that $\left(t_{0}, x\right)$ is Lebesgue point of $u_{1}, u_{2}, f, c$ and $b$. The set $E$ has Lebesgue measure zero. For any fixed $t_{0} \in[0, T] \backslash E$ and $\rho>0$ it follows from Theorem 3.2 that

$$
\int_{B_{\rho}}\left|u_{i}\left(t_{0}, x\right)-u_{i_{0}}(x)\right| \mathrm{d} x \leq \int_{B_{\rho}}\left|u_{i}\left(t_{0}, x\right)-u_{i}^{\epsilon_{m}}\left(t_{0}, x\right)\right| \mathrm{d} x+\omega_{\rho}^{u_{i}}\left(t_{0}\right), \quad i=1,2 .
$$

The pointwise convergence of $\left\{u_{i}^{\epsilon_{m}}\right\}, i=1,2$, yields

$$
\int_{B_{\rho}}\left|u_{i}\left(t_{0}, x\right)-u_{i_{0}}(x)\right| \mathrm{d} x \leq \omega_{\rho}^{u_{i}}\left(t_{0}\right), \quad i=1,2 .
$$

The properties of $\omega_{\rho}^{u_{i}}\left(t_{0}\right), i=1,2$, give (3.63) since $u_{i}$ has compact support. Similarly we obtain relations (3.64)-3.66).

\section{Numerical Results}

\subsection{Non-dimensionalisation of the model}

In this section, we investigate numerically the type of patterns exhibited by model (2.10) in the one-dimensional case. Let $R_{s}>0$ be the cells sensing radius (i.e. the 
maximum range over which cells can detect other surrounding cells). We consider a bounded domain $\Omega=\left[0, R_{s}\right]$, and following the approach in Ref. 24, we choose the non-local terms $F_{i}[\underline{u}, f, c, b], i=1,2$, to be given by

$$
F_{i}[\underline{u}, f, c, b](t, x):=\frac{1}{R_{s}} \int_{0}^{R_{s}} \sum_{k=0}^{1} \eta(k) K(r) g_{i}(\underline{v}(t, x+r \eta(k)), c(t, x), b(t, x)) \mathrm{d} r,
$$

where $\eta(k)=(-1)^{k}, k=0,1$ and $g_{i}, i=1,2$, as described in Sec. 2 (see relation (2.5)).

Let us define the kernel $K$, assuming that it is attractive at medium/long ranges (i.e. at the edges of the cell) and repulsive at very short ranges (i.e. over cell surface), and thus can be defined as

$$
K(x):=q_{a} K_{a}(x)-q_{r} K_{r}(x),
$$

with $q_{a}$ and $q_{r}$ describing the magnitudes of attractive and repulsive interactions, respectively, and $K_{a}(x)$ and $K_{r}(x)$ describe the spatial ranges over which these interactions take place. We consider translated Gaussian attraction and repulsion kernels (as in Ref. 20):

$$
K(x)=\frac{q_{a}}{\sqrt{2 \pi m_{a}^{2}}} e^{-\frac{\left(x-s_{a}\right)^{2}}{2 m_{a}^{2}}}-\frac{q_{r}}{\sqrt{2 \pi m_{r}^{2}}} e^{-\frac{\left(x-s_{r}\right)^{2}}{2 m_{r}^{2}}},
$$

where $s_{a}$ and $s_{r}$ represent half of the length of attraction and repulsion ranges, respectively, with $s_{r}<s_{a}$. Also, $m_{j}=s_{j} / 8, j=a, r$, represent the width of the attractive and the repulsive interaction ranges.

To perform numerical simulations, we first non-dimensionalise system (2.10) by using the following quantities:

$$
\begin{aligned}
\tilde{t} & =\frac{t}{\tau}, \quad \tilde{x}=\frac{x}{L_{0}}, \quad \tilde{u}_{i}=\frac{u_{i}}{k_{u}}, \quad \tilde{f}=\frac{f}{f_{m}}, \\
\tilde{c} & =\frac{c}{c_{m}}, \quad \tilde{b}=\frac{b}{b_{m}}, \quad \tilde{R}_{s}=\frac{R_{s}}{L_{0}}, \quad \tilde{r}=\frac{r}{L_{0}}, \\
\tilde{S}(\tilde{c}, \tilde{b}) & =\frac{\tau k_{u}}{L_{0}^{2}} S\left(c_{m} \tilde{c}+b_{m} \tilde{b}\right), \quad \tilde{S}_{i}(\tilde{c}, \tilde{b})=\frac{\tau k_{u}}{L_{0}^{2}} S_{i}\left(c_{m} \tilde{c}+b_{m} \tilde{b}\right), \\
\tilde{C}_{i}(\tilde{c}, \tilde{b}) & =\frac{\tau f_{m}}{L_{0}^{2}} C_{i}\left(c_{m} \tilde{c}+b_{m} \tilde{b}\right), \quad i=1,2 .
\end{aligned}
$$

The length scale, $L_{0}$, is in the range of $0.1-1 \mathrm{~cm}$, and is defined as the maximum invasion distance of the cancer cells at the early stage of invasion.2 The time scale is defined as $\tau:=L_{0}^{2} / D_{\tau}$, where $D_{\tau}$ is the characteristic diffusion coefficient $\left(\sim 10^{-6} \mathrm{~cm}^{2} \mathrm{~s}^{-1}\right)$. Furthermore, we rescale the cancer cells, the ECM, the integrins and the TGF- $\beta$ with $k_{u}, f_{m}, c_{m}$ and $b_{m}$, respectively. Here, $k_{u}$ is the carrying capacity of the cancer cell populations and it is taken to be $\sim 6.7 \cdot 10^{7}$ cell/volume, and $f_{m}$ is the maximum ECM density at which the ECM fills up all available physical space and it is taken to be equal to $4 \mathrm{mg} /$ volume, as in Ref. 19. Finally, $c_{m}$ 
is the maximum integrin density and it is taken to be $5 \cdot 10^{4}$ integrins per cell (as in Ref. (7), while $b_{m}$ is the maximum TGF- $\beta$ concentration taken to be equal to $141.59 \mathrm{ng} /$ volume (as in Ref. 34).

We choose the dimensionless functions $\tilde{K}(\tilde{r}):=L_{0} K\left(L_{0} \tilde{r}\right)=L_{0} K(r)$ and $\tilde{g}_{i}(\underline{\tilde{u}}, \tilde{f}, \tilde{c}, \tilde{b}):=\left(\tau / L_{0}^{2}\right) g_{i}(\underline{u}, f, c, b), i=1,2$. Therefore, the non-local terms are given by $\tilde{F}_{i}[\underline{\tilde{u}}, \tilde{f}, \tilde{c}, \tilde{b}]:=\left(\tau / L_{0}\right) F_{i}[\underline{u}, f, c, b], i=1,2$.

Finally, we obtain the dimensionless parameters:

$$
\begin{gathered}
\tilde{D}_{u}=\frac{D_{u}}{D_{\tau}}, \quad \tilde{D}_{b}=\frac{D_{b}}{D_{\tau}}, \quad \tilde{M}=\tau M, \quad \tilde{\alpha}=\tau \alpha k_{u}, \quad \tilde{\beta}=\tau \beta k_{u}, \\
\tilde{\theta}_{\beta}=\tau \theta_{\beta} b_{m}, \quad \tilde{\delta}=\tau \delta, \quad \tilde{p}_{3}=\tau p_{3} b_{m}, \quad \tilde{q}=\tau q, \quad \tilde{q}_{b}=\tau q_{b}, \\
\tilde{r}_{i}=\tau r_{i}, \quad \tilde{p}_{i}=\frac{\tau p_{i} k_{u}}{c_{m}} \quad \text { and } \quad \tilde{\mu}_{i}=\frac{\tau \mu_{i} k_{u}}{b_{m}}, \quad i=1,2 .
\end{gathered}
$$

After dropping the tildes for notational convenience, we obtain the following nondimensionalised system:

$$
\begin{aligned}
\frac{\partial u_{1}}{\partial t}= & D_{u} \frac{\partial^{2} u_{1}}{\partial x^{2}}-\frac{\partial}{\partial x}\left(u_{1} F_{1}[\underline{u}, f, c, b]\right) \\
& -M u_{1}+r_{1} u_{1}\left(1-u_{1}-u_{2}\right)\left(1-c_{b} b\right), \\
\frac{\partial u_{2}}{\partial t}= & -\frac{\partial}{\partial x}\left(u_{2} F_{2}[\underline{u}, f, c, b]\right)+M u_{1}+r_{2} u_{2}\left(1-u_{1}-u_{2}\right)\left(1+c_{b} b\right), \\
\frac{\partial f}{\partial t}= & -\alpha u_{1} f-\beta u_{2} f+\theta_{\beta} b f+\delta f(1-f), \\
\frac{\partial c}{\partial t}= & p_{1} u_{1}+p_{2} u_{2}+p_{3} b c-q c \\
\frac{\partial b}{\partial t}= & D_{b} \frac{\partial^{2} b}{\partial x^{2}}+\mu_{1} u_{1}+\mu_{2} u_{2}-q_{b} b .
\end{aligned}
$$

\subsection{Pattern formation}

To discretize our model we use a time-splitting approach. We use a Crank-Nicolson scheme to propagate the solution of the diffusion term. Then, we use the NessyahuTadmor scheme ${ }^{53}$ for the time-propagation of the advection terms. Finally, for the time-propagation of the reaction terms we use a fourth-order Runge-Kutta algorithm, where the integrals are further discretised using the Simpson's rule. All simulations are performed on a domain of length $L=10$ with periodic boundary conditions (introduced to approximate the dynamics on an infinite domain). For this reason, the integrals are wrapped-up at the boundaries. The simulations ran for times up to $t=1000$, but for clarity in Figs. 25 we show mainly the dynamics for $t \leq 400$. If the patterns do not reach a steady state before $t=400$, we add inset figures showing the dynamics for $t \leq 1000$. 

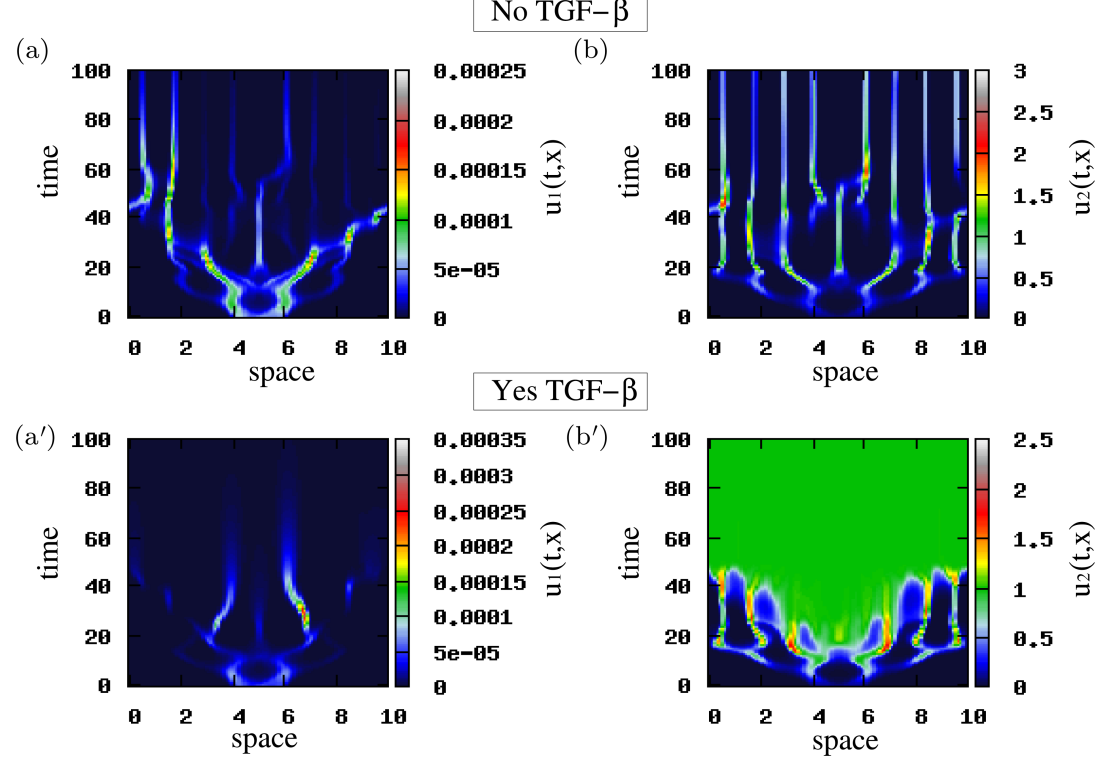

Fig. 2. (Colour online) Patterns exhibited by model 4.6) showing the effect of TGF- $\beta$ on cancer cell density for cell-matrix adhesion greater than cell-cell adhesion, i.e. $s_{1}^{*}=1.8, s_{2}^{*}=0.6, s^{*}=$ $1, c_{1}^{*}=1.9$ and $c_{2}^{*}=2.5$. The rest of model parameters are given in Table A.2. (a), (b) Density of $u_{1}$ and $u_{2}$ populations in the absence of TGF- $\beta ;\left(a^{\prime}\right),\left(b^{\prime}\right)$ Density of $u_{1}$ and $u_{2}$ populations in the presence of TGF- $\beta$.

The initial conditions for the cancer cell populations are small random perturbations of rectangular-shaped aggregations located in the middle of the domain

$$
u_{i}(0, x)= \begin{cases}u_{i}^{c}+\operatorname{rand}\left(0,10^{-4}\right), & x \in(L / 2-1, L / 2+1), \\ 0, & \text { everywhere else }\end{cases}
$$

with $u_{1}^{c}=0$ and $u_{2}^{c}=0.1$. For the ECM density, $f$, we assume that the tumour has already degraded some of its surrounding tissues:

$$
f(0, x)=1-0.5 u_{1}(0, x)-0.5 u_{2}(0, x) .
$$

Finally, the integrin density and TGF- $\beta$ concentration, $c$ and $b$, respectively, are proportional to the initial tumour cell density

$$
c(0, x)=0.5 u_{1}(0, x)+0.5 u_{2}(0, x)
$$

and

$$
b(0, x)=0.05 u_{1}(0, x)+0.05 u_{2}(0, x) .
$$

To investigate the effect of TGF- $\beta$ signalling on cell proliferation, movement and aggregation (the last two aspects being controlled by cell adhesion), we focus on three possible cases for the magnitudes of cell-cell and cell-matrix adhesion. For each of these three cases, we investigate the dynamics of $u_{1}$ and $u_{2}$ populations 
when TGF- $\beta$ is absent and does not influence cell proliferation or cell adhesion (i.e. for $\left.c_{b}=a_{b_{i}}=d_{b}=e_{b_{i}}=\theta_{\beta}=p_{3}=\mu_{1}=\mu_{2}=0, i=1,2\right)$, and when TGF- $\beta$ is present and influences both cell proliferation and cell adhesion (i.e. for $c_{b}=20$ and $\left.a_{b_{i}}, d_{b}, e_{b_{i}}, \theta_{\beta}, p_{3}, \mu_{1}, \mu_{2}, \neq 0, i=1,2\right)$.

(i) Cell-cell adhesion $<$ cell-matrix adhesion. To investigate the effect of greater cell-matrix adhesion, we choose $s_{1}^{*}=1.8, s_{2}^{*}=0.6, s^{*}=1, c_{1}^{*}=1.9$ and $c_{2}^{*}=2.5$ and the rest of model parameters as given in Table A.2. We see in Figs. 2(a) and 2(b) that in the absence of TGF- $\beta$, the population of early stage cancer cells $\left(u_{1}\right)$ decreases, while the population of late stage cancer cells $\left(u_{2}\right)$ increases and dominates the long-term dynamics. This behaviour is expected due to the mutation term "- $M u_{1}$ ", and due to large cell-matrix adhesion, which impedes cells to move and thus leads to the formation of stationary pulses for $t>50$. Considering now the effect of TGF- $\beta$, we see in Figs. 2 $\left(\mathrm{a}^{\prime}\right)$ and $2\left(\mathrm{~b}^{\prime}\right)$ that population $u_{1}$ vanishes faster, due to the presence of antiproliferative and proapoptotic signals from TGF- $\beta$ (described by $c_{b}>0$ in Eq. (4.6a) ). Population $u_{2}$ persists and increases significantly, due to the promoting effects of TGF- $\beta$ on the late stages of cancer, which also induces the movement of the cancer cells (via EMT) thus leading to their spread over the domain until they reach the boundaries.

(a)
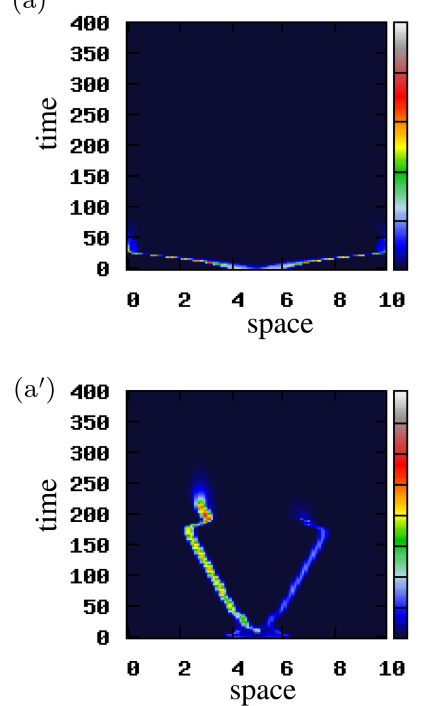

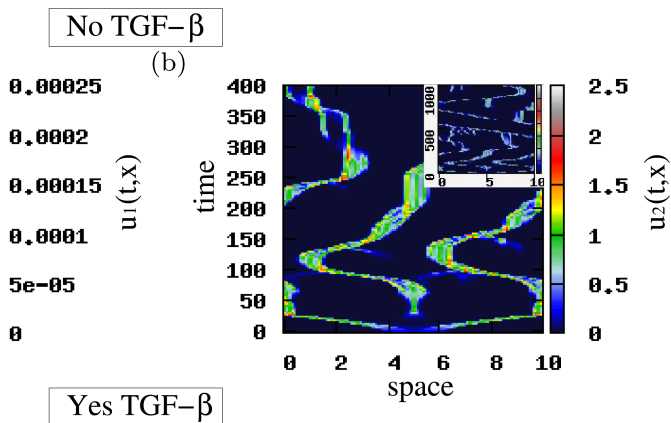

Yes TGF- $\beta$

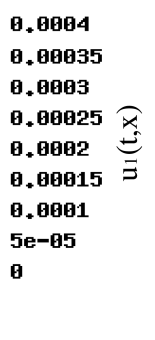

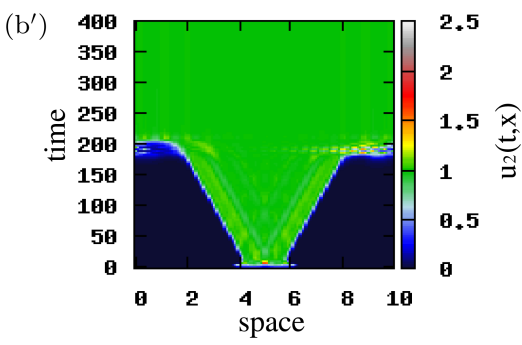

Fig. 3. (Colour online) Patterns exhibited by model (4.6) showing the effect of TGF- $\beta$ on cancer cell density for cell-cell adhesion greater than cell-matrix adhesion, i.e. $s_{1}^{*}=2.4, s^{*}=2.1, s_{2}^{*}=$ $2, c_{1}^{*}=1.1$ and $c_{2}^{*}=0.9$. The rest of model parameters are given in Table A.2. (a), (b) Density of $u_{1}$ and $u_{2}$ populations in the absence of TGF- $\beta$. The inset in panel (b) shows the long-term dynamics of $u_{2}(t, x)$ (for $\left.t \leq 1000\right) ;\left(\mathrm{a}^{\prime}\right),\left(\mathrm{b}^{\prime}\right)$ Density of $u_{1}$ and $u_{2}$ populations in the presence of TGF- $\beta$. 
(ii) Cell-cell adhesion $>$ cell-matrix adhesion. To investigate the effect of greater cell-cell adhesion, we choose $s_{1}^{*}=2.4, s^{*}=2.1, s_{2}^{*}=2, c_{1}^{*}=1.1$ and $c_{2}^{*}=0.9$, and the rest of model parameters as given in Table A.2. We see in Figs. 3 (a) and 3 (b) that due to the weak cell-matrix adhesive forces, $u_{1}$ and $u_{2}$ cells start to move through the domain in a collective manner. Figures 3 (a) and $3\left(a^{\prime}\right)$ show that $u_{1}$ population vanishes in the absence and in the presence of TGF- $\beta$ (due to the mutation term). We also note that the spread of $u_{1}$ cells is reduced in the presence of TGF- $\beta$, likely due to the positive effect of TGF- $\beta$ on cell-matrix adhesion (see the term " $+e_{b_{i}} b$ " in Eqs. (2.6)). In Figs. 3(b) and 3(b') we see that the $u_{2}$ population changes its movement from a chaotic-like dynamics (in the absence of TGF- $\beta$; panel (b)) to a spread over the whole domain (in the presence of TGF- $\beta$; panel $\left.\left(b^{\prime}\right)\right)$, as a result of a decrease in the cell-cell adhesion induced by the tumour growth factor.

(iii) Cell-cell adhesion $=$ cell-matrix adhesion . To ensure the same values for the adhesive strength functions (2.6) when there is no TGF- $\beta$ in the system, we choose $s_{i}^{*}=s^{*}=c_{i}^{*}=0.8$ and $a_{i}=d=e_{i}=0.5, i=1,2$. In Figs. 4(a) and $4(b)$, we see that some cells in the two cancer sub-populations move quickly to the left and the right, reaching the boundaries, while other cells (both $u_{1}$ and $u_{2}$ ) stay in the middle of the domain and create a chaotic-like pattern (even if $u_{1}$ is slowly eliminated for $t>50$ ). If we now add TGF- $\beta$ to the system, we see
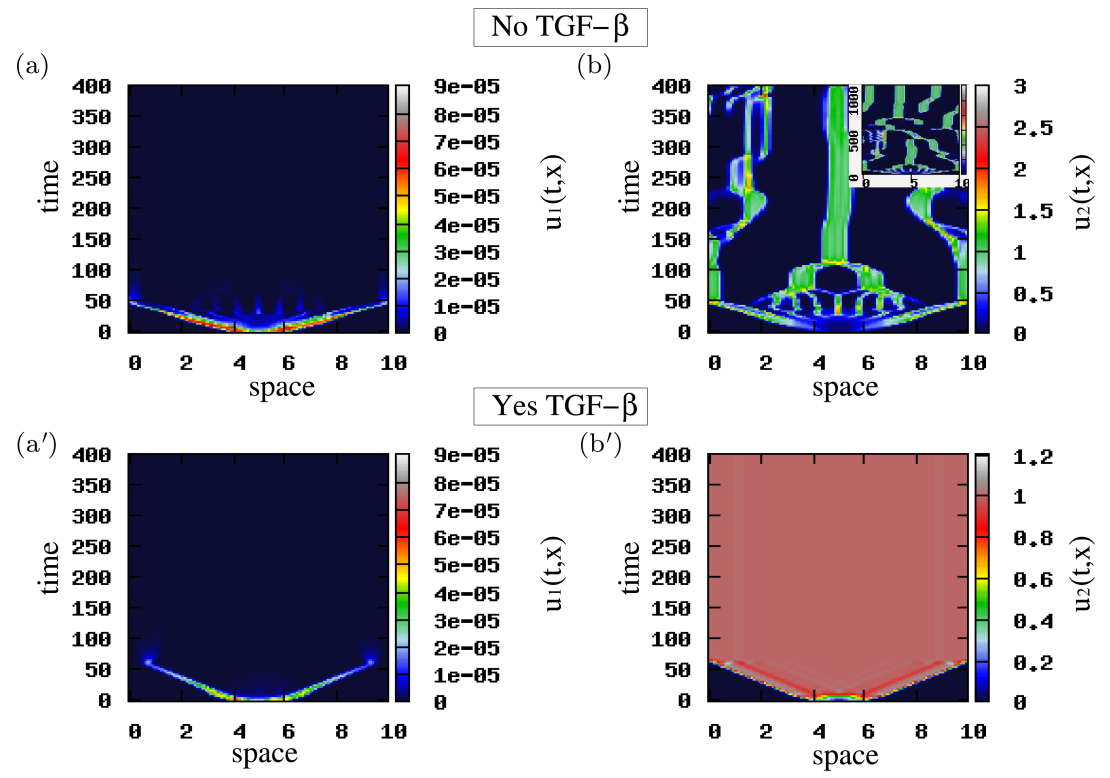

Fig. 4. (Colour online) Patterns exhibited by model (4.6) showing the effect of TGF- $\beta$ on cancer cell density for the same cell-cell and cell-matrix adhesion, i.e. $s_{i}^{*}=s^{*}=c_{i}^{*}=0.8$ and $a_{i}=d=$ $e_{i}=0.5, i=1,2$. The rest of model parameters are given in Table A.2. (a), (b) Density of $u_{1}$ and $u_{2}$ populations in the absence of TGF- $\beta$. The inset in panel (b) shows the long-term dynamics of $u_{2}(t, x)$ (for $\left.t \leq 1000\right) ;\left(\mathrm{a}^{\prime}\right),\left(\mathrm{b}^{\prime}\right)$ Density of $u_{1}$ and $u_{2}$ populations in the presence of TGF- $\beta$. 
in Figs. $4\left(\mathrm{a}^{\prime}\right)$ and $4\left(\mathrm{~b}^{\prime}\right)$ that both $u_{1}$ and $u_{2}$ populations move slower towards the edges of the domain. In contrast to the $u_{2}$ population in the absence of TGF- $\beta$, which exhibits a chaotic clumping and splitting behaviour (panel (b)), the $u_{2}$ population in the presence of TGF- $\beta$ exhibits travelling-wave dynamics (panel $\left(b^{\prime}\right)$ ). This is different from the dynamics observed in Fig. 3 $\left(b^{\prime}\right)$ where the $u_{2}$ cells move in a travelling-wave manner up to $t=200$, after which they quickly move towards the boundaries.

Reducing now the magnitudes of cellular adhesion forces to $s_{i}^{*}=s^{*}=c_{i}^{*}=$ $0.1, i=1,2$, we see in Fig. [5 that irrespective of the absence/presence of TGF- $\beta$, population $u_{1}$ forms a stationary aggregation that eventually vanishes for large times, while population $u_{2}$ exhibits a travelling wave. This behaviour might be explained by the combined effect of high mutation rate and clonal competition (see the logistic growth terms in (2.2)), since adhesive forces are very small and lead to the spread of population $u_{2}$. In contrast to the dynamics in Figs. 3(b) and [3( $\left.\mathrm{b}^{\prime}\right)$, and $4(\mathrm{~b})$ and $4\left(\mathrm{~b}^{\prime}\right)$, where the $u_{2}$ cells seem to travel slower towards the boundaries in the presence of TGF- $\beta$ (compared to the absence of TGF- $\beta$ ), in Fig. 5 the $u_{2}$ cells travel faster to the boundaries in the presence of TGF- $\beta$. We deduce from here that the spread of tumour cells depends both on the magnitude of adhesive forces as well as on the presence of TGF- $\beta$ molecules.

(a)

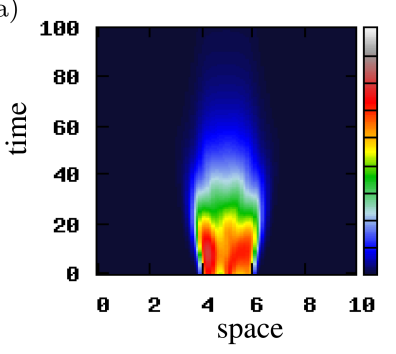

\section{No TGF- $\beta$}
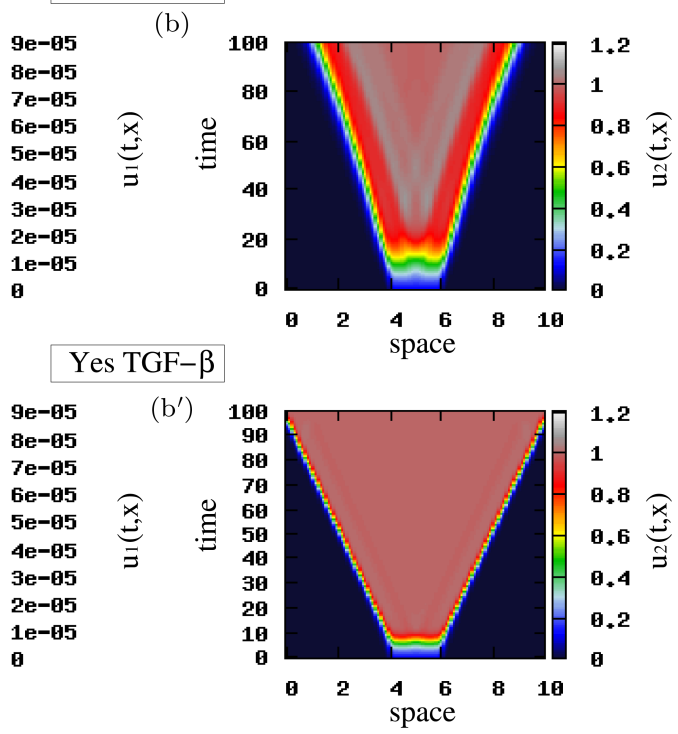

Fig. 5. (Colour online) Patterns exhibited by model (4.6) showing the effect of TGF- $\beta$ on cancer cell density for the same cell-cell and cell-matrix adhesion, i.e. $s_{i}^{*}=s^{*}=c_{i}^{*}=0.1$ and $a_{i}=d=$ $e_{i}=0.5, i=1,2$. The rest of model parameters are given in Table A.2. (a), (b) Density of $u_{1}$ and $u_{2}$ populations in the absence of TGF- $\beta$; $\left(\mathrm{a}^{\prime}\right),\left(\mathrm{b}^{\prime}\right)$ Density of $u_{1}$ and $u_{2}$ populations in the presence of TGF- $\beta$. 
We note here that we also investigated numerically the case when TGF$\beta$ is present, but does not influence cell proliferation or cell adhesion (i.e. for $\theta_{\beta}, p_{3}, \mu_{1}, \mu_{2} \neq 0$, and $\left.c_{b}=a_{b_{i}}=d_{b}=e_{b_{i}}=0, i=1,2\right)$. The patterns (not shown here) that we obtained were similar to those presented in Figs. 2(a) and 2(b), 3(a) and 33(b), 4(a) and 4(b), 5(a) and 5)(b). This suggests that the effect of TGF- $\beta$ on the cancer cell density is greater than the effect on the ECM and on the integrins density.

\section{Conclusion and Discussion}

In this paper, we introduced a model of integro-differential equations describing the dynamics of early stage and late stage cancer cell populations, under the effect of TGF- $\beta$ signalling. The model was then used to investigate the role of TGF- $\beta$ on cellular adhesion and proliferation.

We first proved the global existence of bounded solutions to our non-local model by taking a vanishing viscosity approach and approximating our model with a nonlocal parabolic PDE. The proof used the Banach contraction mapping theorem, the Möser-Alikakos method and the vanishing viscosity method.

We then investigated numerically the solution of this non-local model, paying particular attention to the effect of TGF- $\beta$ on cell-cell and cell-matrix interactions. We showed that: (i) In the absence of TGF- $\beta$, the magnitudes of cell-cell and cellmatrix interactions influenced the formation of cancer cell aggregation at specific position in space (see Figs. 2(b), 3)(b) and 4(b)); (ii) The consideration of TGF- $\beta$ leads to the spread of mutated (i.e. $u_{2}$ ) cancer cells over the whole domain mainly in a travelling-wave manner (with no cell aggregations; see Figs. [3( $\left(b^{\prime}\right), 4\left(b^{\prime}\right)$ and $\left.5\left(b^{\prime}\right)\right)$. We also emphasise that the speed at which cells spread depended on the presence/absence of TGF- $\beta$ and on the magnitudes of cell adhesion forces (see Figs. 4(b) and $4\left(b^{\prime}\right)$ versus Figs. $5(b)$ and $\left.5\left(b^{\prime}\right)\right)$.

While the numerical investigation of cancer spread uncovered an interesting combined effect of cell adhesion and presence/absence of TGF- $\beta$, in the future we plan to investigate analytically the travelling waves and study the effect of parameters related to TGF- $\beta$ and cell adhesion on the speed of these waves.

\section{Appendix A. Summary of Model Variables and Parameters}

Here we present two tables with the model variables and parameters. In Table A.1, we list the model variables with their units. In Table A.2, we list the parameters of our model and their corresponding units and non-dimensional values used in the simulations.

\section{Parameter estimation}

- Attraction and repulsion ranges were chosen to be smaller or equal to sensing radius, with the repulsion range to be smaller than the attraction range.25 
Table A.1. A list of model variables with their units. Since we are in $1 \mathrm{D}$, length and volume coincide and we express the variables in terms of domain length.

\begin{tabular}{clc}
\hline Variable & \multicolumn{1}{c}{ Description } & Dimensional units \\
\hline$u_{1}$ & Early stage cancer cell density & cell/length \\
$u_{2}$ & Late stage cancer cell density & cell/length \\
$f$ & ECM density & mg/length \\
$c$ & Integrin density & integrins $/$ cell \\
$b$ & TGF- $\beta$ concentration & mg/length \\
\hline
\end{tabular}

Table A.2. A list of model parameters with their units and their non-dimensional values, obtained from 4.4 and 4.5, which we used during numerical simulations.

\begin{tabular}{|c|c|c|c|c|}
\hline Param. & Description & Dimensional units & Non-dim. value $(\tilde{p})$ & Reference \\
\hline$D_{u}$ & Diffusion coefficient of $u_{1}$ & length $^{2} /$ time & 0.0001 & 11 \\
\hline$R_{s}$ & Sensing radius & length & 0.99 & 3 and 24 \\
\hline$q_{a}$ & Magnitude of attraction & length $^{2} /$ cell & 0.09 & Estimated \\
\hline$q_{r}$ & Magnitude of repulsion & $\operatorname{length}^{2} /$ cell & 0.01 & Estimated \\
\hline$s_{a}$ & Attraction range & length & 0.99 & Estimated \\
\hline$s_{r}$ & Repulsion range & length & 0.25 & Estimated \\
\hline$m_{a}$ & $\begin{array}{l}\text { Width of attraction } \\
\text { kernel }\end{array}$ & length & $0.99 / 8$ & Estimated \\
\hline$m_{r}$ & Width of repulsion kernel & length & $0.25 / 8$ & Estimated \\
\hline$r_{1}$ & Growth rate of $u_{1}$ & $1 /$ time & 0.1 & 50 \\
\hline$r_{2}$ & Growth rate of $u_{2}$ & $1 /$ time & 0.2 & 50 \\
\hline$M$ & Mutation rate & 1/time & 0.05 & 14,28 and 43 \\
\hline$c_{b}$ & $\begin{array}{l}\text { Coeff. related to the } \\
\text { effect of TGF- } \beta \text { on } \\
\text { cancer cell proliferation }\end{array}$ & Nondim. & 20 & Estimated \\
\hline$a_{1}$ & $\begin{array}{l}\text { Coeff. related to the } \\
\text { number of integrins } \\
\text { necessary for max } \\
\text { self-adhesion between } \\
u_{1}\end{array}$ & cell/integrins & 0.7 & Estimated \\
\hline$a_{2}$ & $\begin{array}{l}\text { Coeff. related to the } \\
\text { number of integrins } \\
\text { necessary for max } \\
\text { self-adhesion between } \\
u_{2}\end{array}$ & cell/integrins & 0.3 & Estimated \\
\hline$d$ & $\begin{array}{l}\text { Coeff. related to the } \\
\text { number of integrins } \\
\text { necessary for max } \\
\text { cell-cell cross-adhesion }\end{array}$ & cell/integrins & 0.5 & Estimated \\
\hline$e_{1}$ & $\begin{array}{l}\text { Coeff. related to the } \\
\text { number of integrins } \\
\text { necessary for max } \\
\text { cell-ECM adhesion for } \\
u_{1}\end{array}$ & cell/integrins & 1.8 & Estimated \\
\hline$e_{2}$ & $\begin{array}{l}\text { Coeff. related to the } \\
\text { number of integrins } \\
\text { necessary for max } \\
\text { cell-ECM adhesion for } \\
u_{2}\end{array}$ & cell/integrins & 2.5 & Estimated \\
\hline
\end{tabular}


Table A.2. (Continued)

\begin{tabular}{|c|c|c|c|c|}
\hline Param. & Description & Dimensional units & Non-dim. value $(\tilde{p})$ & Reference \\
\hline$a_{b_{1}}$ & $\begin{array}{l}\text { Coeff. related to the } \\
\text { effect of TGF- } \beta \text { on } \\
\text { self-adhesion between } \\
u_{1} \text { cells }\end{array}$ & length/mg & 0.5 & Estimated \\
\hline$a_{b_{2}}$ & $\begin{array}{l}\text { Coeff. related to the } \\
\text { effect of TGF- } \beta \text { on } \\
\text { self-adhesion between } \\
u_{2} \text { cells }\end{array}$ & length $/ \mathrm{mg}$ & 0.3 & Estimated \\
\hline$d_{b}$ & $\begin{array}{l}\text { Coeff. related to the } \\
\text { effect of TGF- } \beta \text { on } \\
\text { cell-cell cross-adhesion }\end{array}$ & length $/ \mathrm{mg}$ & 0.4 & Estimated \\
\hline$e_{b_{1}}$ & $\begin{array}{l}\text { Coeff. related to the } \\
\text { effect of TGF- } \beta \text { on } \\
\text { cell-ECM adhesion for } \\
u_{1} \text { cells }\end{array}$ & length/mg & 0.8 & Estimated \\
\hline$e_{b_{2}}$ & $\begin{array}{l}\text { Coeff. related to the } \\
\text { effect of TGF- } \beta \text { on } \\
\text { cell-ECM adhesion for } \\
u_{2} \text { cells }\end{array}$ & length $/ \mathrm{mg}$ & 0.9 & Estimated \\
\hline$s_{1}^{*}$ & $\begin{array}{l}\text { Magnitude of } \\
\text { self-adhesion forces of } \\
u_{1}\end{array}$ & length $/($ time $\cdot$ cell $)$ & $0.1-2.4$ & Estimated \\
\hline$s_{2}^{*}$ & $\begin{array}{l}\text { Magnitude of } \\
\text { self-adhesion forces of } \\
u_{2}\end{array}$ & length $/($ time $\cdot$ cell $)$ & $0.1-2$ & Estimated \\
\hline$s^{*}$ & $\begin{array}{l}\text { Magnitude of } \\
\text { cross-adhesion forces }\end{array}$ & length/(time $\cdot$ cell $)$ & $0.1-2.1$ & Estimated \\
\hline$c_{1}^{*}$ & $\begin{array}{l}\text { Magnitude of cell-ECM } \\
\text { forces of } u_{1}\end{array}$ & length $/($ time $\cdot$ cell $)$ & $0.1-1.9$ & Estimated \\
\hline$c_{2}^{*}$ & $\begin{array}{l}\text { Magnitude of cell-ECM } \\
\text { forces of } u_{2}\end{array}$ & length/(time $\cdot$ cell $)$ & $0.1-2.5$ & Estimated \\
\hline$\alpha$ & $\begin{array}{l}\text { Rate of ECM } \\
\quad \text { degradation by } u_{1}\end{array}$ & length/(time $\cdot$ cell $)$ & 1 & 59 \\
\hline$\beta$ & $\begin{array}{l}\text { Rate of ECM } \\
\quad \text { degradation by } u_{2}\end{array}$ & length $/($ time $\cdot$ cell $)$ & 2 & 59 \\
\hline$\theta_{\beta}$ & $\begin{array}{l}\text { Binding rate of TGF- } \beta \\
\text { to ECM components }\end{array}$ & length $/($ time $\cdot \mathrm{mg})$ & 0.77 & Estimated \\
\hline$\delta$ & ECM remodelling rate & $1 /$ time & 0.25 & 11 \\
\hline$p_{1}$ & $\begin{array}{l}\text { Production rate of } c \text { by } \\
u_{1}\end{array}$ & integrins $/($ time $\cdot$ cell $)$ & 0.05 & Estimated \\
\hline$p_{2}$ & $\begin{array}{l}\text { Production rate of } c \text { by } \\
u_{2}\end{array}$ & integrins $/($ time $\cdot$ cell $)$ & 0.1 & Estimated \\
\hline$p_{3}$ & $\begin{array}{l}\text { Up-regulation rate of } c \\
\text { by } b\end{array}$ & length $/($ time $\cdot \mathrm{mg})$ & 0.2 & Estimated \\
\hline$q$ & Decay rate of $c$ & $1 /$ time & 0.3 & 39 \\
\hline$D_{b}$ & $\begin{array}{l}\text { Diffusion coefficient of } \\
\text { TGF- } \beta\end{array}$ & length $^{2} /$ time & 0.007 & 64 \\
\hline$\mu_{1}$ & $\begin{array}{l}\text { Production rate of } b \text { by } \\
u_{1}\end{array}$ & $\mathrm{mg} /($ time $\cdot$ cell $)$ & 0.05 & 36 and 69 \\
\hline$\mu_{2}$ & $\begin{array}{l}\text { Production rate of } b \text { by } \\
u_{2}\end{array}$ & $\mathrm{mg} /($ time $\cdot$ cell $)$ & 0.05 & 36 and 69 \\
\hline$q_{b}$ & Decay rate of $b$ & $1 /$ time & 0.05 & 39 \\
\hline
\end{tabular}


- Various experimental studies $\stackrel{15[50}{ }$ have shown that doubling times for tumour cells range from 1-10 days. This corresponds to growth rates between $(\ln (2) / 10, \ln (2) / 1)=(0.07,0.7)$. In this study, we assume that $\tilde{r}_{1}=0.1$ and $\tilde{r}_{2}=0.2$.

- Experimental studies $\sqrt{14 \mid 28,43}$ have shown that the mutation rate ranges between $M=10^{-3} /$ day and $M=0.1 /$ day. Thus the non-dimensional value of the mutation rate is in the range between $\tilde{M}=0.001$ and $\tilde{M}=0.1$ (for highly aggressive tumours). In this study, we choose $\tilde{M}=0.05$.

- The parameters $a_{i}, d, e_{i}, s_{i}^{*}, s^{*}, c_{i}^{*}, i=1,2$, were based on the range of the adhesion strength parameters used in Ref. 3

- Experimental studies ${ }^{35}$ have shown greater production of integrins for mutated cancer cells. Thus, we choose $p_{1}<p_{2}$.

- Experimental studies 16 17/39/40 have shown that the half-lifes of the integrins range from 0.04-4 days. This corresponds to a decay rate between $(\ln (2) / 4, \ln (2) / 0.04)=(0.17,17.3)$. In this study, we assume that $\tilde{q}=0.3$.

- The remodelling rate was chosen to be greater than cell proliferation rate, as considered also in Ref. 11.

\section{Acknowledgments}

The authors would like to thank Dr. D. Trucu for fruitful discussions. V.B. acknowledges support from an Engineering and Physical Sciences Research Council (UK) Grant Number EP/L504932/1. R.E. was partially supported by an Engineering and Physical Sciences Research Council (UK) Grant Number EP/K033689/1.

\section{References}

1. D. Ambrosi and L. Preziosi, On the closure of mass balance models for tumor growth, Math. Models Methods Appl. Sci. 12 (2002) 737-754.

2. A. R. A. Anderson, M. A. J. Chaplain, E. L. Newman, R. J. C. Steele and A. M. Thompson, Mathematical modelling of tumour invasion and metastasis, J. Theor. Med. 2 (2000) 129-154.

3. N. J. Armstrong, K. J. Painter and J. A. Sherratt, A continuum approach to modelling cell-cell adhesion, J. Theor. Biol. 243 (2006) 98-113.

4. G. Ascolani and P. Liò, Modeling TGF- $\beta$ in early stages of cancer tissue dynamics, PLoS One 9 (2014) e88533.

5. N. Bellomo, A. Bellouquid and N. Chouhad, From a multiscale derivation of nonlinear cross-diffusion models to Keller-Segel models in a Navier-Stokes fluid, Math. Models Methods Appl. Sci. 26 (2016) 2041-2069.

6. N. Bellomo, N. Li and P. K. Maini, On the foundations of cancer modelling: Selected topics, speculations and perspectives, Math. Models Methods Appl. Sci. 18 (2008) 593-646.

7. S. Benedetto, R. Pulito, S. G. Crich, G. Tarone, S. Aime, L. Silengo and J. Hamm, Quantification of the expression level of integrin receptor $\alpha \mathrm{v} \beta 3$ in cell lines and MR imaging with antibody-coated iron oxide particles, Magn. Reson. Med. 56 (2006) $711-716$. 
8. H. Byrne and L. Preziosi, Modelling solid tumour growth using the theory of mixtures, Math. Med. Biol. 20 (2003) 341-366.

9. F. Calvo and E. Sahai, Cell communication networks in cancer invasion, Curr. Opin. Cell Biol. 23 (2011) 621-629.

10. M. A. J. Chaplain, M. Lachowicz, Z. Szymańska and D. Wrzosek, Mathematical modelling of cancer invasion: The importance of cell-cell adhesion and cell-matrix adhesion, Math. Models Methods Appl. Sci. 21 (2011) 719-743.

11. M. A. J. Chaplain and G. Lolas, Mathematical modelling of cancer invasion of tissue: Dynamic heterogeneity, Netw. Heterog. Media 1 (2006) 399-439.

12. A. Chapman, L. F. del Ama, J. Ferguson, J. Kamarashev, C. Wellbrock and A. Hurlstone, Heterogeneous tumor subpopulations cooperate to drive invasion, Cell Rep. 8 (2014) 688-695.

13. J. W. Cholewa and T. Dlotko, Global Attractors in Abstract Parabolic Problems, Vol. 278 (Cambridge Univ. Press, 2000).

14. C. Cillo, J. E. Dick, V. Ling and R. P. Hill, Generation of drug-resistant variants in metastatic B16 mouse melanoma cell lines, Cancer Res. 47 (1987) 2604-2608.

15. D. Cunningham and Z. You, In vitro and in vivo model systems used in prostate cancer research, J. Biol. Meth. 2 (2015) e17.

16. T. L. Davis, I. Rabinovitz, B. W. Futscher, M. Schnölzer, F. Burger, Y. Liu, M. KuleszMartin and A. E. Cress, Identification of a novel structural variant of the $\alpha 6$ integrin, J. Biol. Chem. 276 (2001) 26099-26106.

17. M. Delcommenne and C. H. Streuli, Control of integrin expression by extracellular matrix, J. Biol. Chem. 270 (1995) 26794-26801.

18. J. J. Deman, L. C. Vakaet and E. A. Bruyneel, Cell size and mutual cell adhesion. II. Evidence for a relation between cell size, long-range electrostatic repulsion and intercellular adhesiveness during density-regulated growth in suspension, J. Membr. Biol. 26 (1976) 205-215.

19. P. Domschke, D. Trucu, A. Gerisch and M. A. J. Chaplain, Mathematical modelling of cancer invasion: Implications of cell adhesion variability for tumour infiltrative growth patterns, J. Theor. Biol. 361 (2014) 41-60.

20. R. Eftimie, G. de Vries, M. A. Lewis and F. Lutscher, Modeling group formation and activity patterns in self-organizing collectives of individuals, Bull. Math. Biol. 69 (2007) 1537-1565.

21. L. C. Evans, Partial Differential Equations, Graduate Studies in Mathematics, Vol. 19 (Amer. Math. Soc., 2010).

22. J. Fan and K. Zhao, A note on a 3D haptotaxis model of cancer invasion, Appl. Math. Res. Exp. 2014 (2014) 74-86.

23. B. Geiger, Long-range morphogenetic signals and cell adhesion, BioEssays 13 (1991) 665-666.

24. A. Gerisch and M. A. J. Chaplain, Mathematical modelling of cancer cell invasion of tissue: Local and non-local models and the effect of adhesion, J. Theor. Biol. 250 (2008) 684-704.

25. J. E. F. Green, S. L. Waters, J. P. Whiteley, L. Edelstein-Keshet, K. M. Shakesheff and H. M. Byrne, Non-local models for the formation of hepatocyte-stellate cell aggregates, J. Theor. Biol. 267 (2010) 106-120.

26. D. Hanahan and R. A. Weinberg, The hallmarks of cancer, Cell 100 (2000) 57-70.

27. D. Henry, Geometric Theory of Semilinear Parabolic Systems, Vol. 840 (SpringerVerlag, 1981).

28. R. P. Hill, A. F. Chambers and V. Ling, Dynamic heterogeneity: Rapid generation of metastatic variants in mouse B16 melanoma cells, Science 224 (1984) 998-1001. 
29. T. Hillen, C. Rohde and F. Lutscher, Existence of weak solutions for a hyperbolic model of chemosensitive movement, J. Math. Anal. Appl. 260 (2001) 173-199.

30. S. Huang and S. Chakrabarty, Regulation of fibronectin and laminin receptor expression, fibronectin and laminin secretion in human colon cancer cells by transforming growth factor- $\beta 1$, Int. J. Cancer 57 (1994) 742-746.

31. B. Kaminska, A. Wesolowska and M. Danilkiewicz, TGF beta signalling and its role in tumour pathogenesis, Acta Biochim. Pol. 52 (2005) 329.

32. E. Kang and J. Lee, Global solutions to chemotaxis-haptotaxis tumor invasion system with tissue re-establishment, J. Chungcheong Math. Soc. 28 (2015) 161-172.

33. L. Khalique, A. Ayhan, M. E. Weale, I. J. Jacobs, S. J. Ramus and S. A. Gayther, Genetic intra-tumour heterogeneity in epithelial ovarian cancer and its implications for molecular diagnosis of tumours, J. Pathol. 211 (2007) 286-295.

34. S. A. Khan, J. Joyce and T. Tsuda, Quantification of active and total transforming growth factor- $\beta$ levels in serum and solid organ tissues by bioassay, BMC Res. Notes $5(2012) 1$.

35. Y. Kidera et al., Reduction of lung metastasis, cell invasion and adhesion in mouse melanoma by statin-induced blockade of the rho/rho-associated coiled-coil-containing protein kinase pathway, J. Exp. Clin. Cancer Res. 29 (2010) 127.

36. Y. Kim and H. G. Othmer, A hybrid model of tumor-stromal interactions in breast cancer, Bull. Math. Biol. 75 (2013) 1304-1350.

37. A. K. Laird, Dynamics of tumor growth, Brit. J. Cancer 18 (1964) 490-502.

38. J. T. Leith, S. Michelson and A. S. Glicksman, Competitive exclusion of clonal subpopulations in heterogeneous tumours after stromal injury, Brit. J. Cancer 59 (1989) $22-27$.

39. J. Liu, X. He, Y. Qi, X. Tian, S. J. Monkley, D. R. Critchley, S. A. Corbett, S. F. Lowry, A. M. Graham and S. Li, Talin1 regulates integrin turnover to promote embryonic epithelial morphogenesis, Molec. Cell Biol. 31 (2011) 3366-3377.

40. V. H. Lobert, A. Brech, N. M. Pedersen, J. Wesche, A. Oppelt, L. Malerød and H. Stenmark, Ubiquitination of $\alpha 5 \beta 1$ integrin controls fibroblast migration through lysosomal degradation of fibronectin-integrin complexes, Dev. Cell 19 (2010) 148-159.

41. K. R. Loeb and L. A. Loeb, Significance of multiple mutations in cancer, Carcinogenesis 21 (2000) 379-385.

42. F. A. Mamuya and M. K. Duncan, aV integrins and TGF- $\beta$-induced EMT: A circle of regulation, J. Cell Molec. Med. 16 (2012) 445-455.

43. M. M. Mareel, P. De Baetselier and F. M. Van Roy, Mechanisms of Invasion and Metastasis (CRC Press, 1991).

44. S. Markowitz et al., Inactivation of the type a TGF- $\beta$ receptor in colon cancer cells with microsatellite instability, Science 268 (1995) 1336-1338.

45. A. Marusyk and K. Polyak, Tumor heterogeneity: Causes and consequences, Biochim. Biophys. Acta 1805 (2010) 105.

46. S. Michelson and J. Leith, Autocrine and paracrine growth factors in tumor growth: A mathematical model, Bull. Math. Biol. 53 (1991) 639-656.

47. K. Miyazono, Transforming growth factor- $\beta$ signaling in epithelial-mesenchymal transition and progression of cancer, P. Jpn. Acad. B-Phys. 85 (2009) 314-323.

48. A. Mogilner and L. Edelstein-Keshet, Selecting a common direction: I. How orientational order can arise from simple contact responses between interacting cells, $J$. Math. Biol. 33 (1995) 619-660.

49. A. Mogilner, L. Edelstein-Keshet and G. B. Ermentrout, Selecting a common direction: II. Peak-like solutions representing total alignment of cell clusters, J. Math. Biol. 34 (1996) 811-842. 
50. F. Morani, S. Phadngam, C. Follo, R. Titone, V. Thongrakard, A. Galetto, O. Alabiso and C. Isidoro, PTEN deficiency and mutant p53 confer glucose-addiction to thyroid cancer cells: Impact of glucose depletion on cell proliferation, cell survival, autophagy and cell migration, Genes Cancer 5 (2014) 226-239.

51. A. Moustakas and C.-H. Heldin, Signaling networks guiding epithelial-mesenchymal transitions during embryogenesis and cancer progression, Cancer Sci. 98 (2007) 15121520 .

52. A. Nawshad, D. LaGamba, A. Polad and E. D. Hay, Transforming growth factor- $\beta$ signaling during epithelial-mesenchymal transformation: Implications for embryogenesis and tumor metastasis, Cells Tissues Organs 179 (2005) 11-23.

53. H. Nessyahu and E. Tadmor, Non-oscillatory central differencing for hyperbolic conservation laws, J. Comput. Phys. 87 (1990) 408-463.

54. G. L. Nicholson, Tumor cell instability, diversification and progression to the metastatic phenotype: From oncogene to oncofetal expression, Cancer Res. 47 (1987) 1473-1487.

55. N. Outada, N. Vauchelet, T. Akrid and M. Khaladi, From kinetic theory of multicellular systems to hyperbolic tissue equations: Asymptotic limits and computing, Math. Models Methods Appl. Sci. 26 (2016) 2709-2734.

56. K. J. Painter, N. J. Armstrong and J. A. Sherratt, The impact of adhesion on cellular invasion processes in cancer and development, J. Theor. Biol. 264 (2010) 1057-1067.

57. D. R. Principe, J. A. Doll, J. Bauer, B. Jung, H. G. Munshi, L. Bartholin, B. Pasche, C. Lee and P. J. Grippo, TGF- $\beta$ : Duality of function between tumor prevention and carcinogenesis, J. Natl. Cancer I. 106 (2014) djt369.

58. T. Runst and W. Sickel, Sobolev Spaces of Fractional Order, Nemytskij Operators, and Nonlinear Partial Differential Equations, Vol. 3 (Walter de Gruyter, 1996).

59. J. A. Sherratt, S. A. Gourley, N. J. Armstrong and K. J. Painter, Boundedness of solutions of a non-local reaction-diffusion model for adhesion in cell aggregation and cancer invasion, Eur. J. Appl. Math. 20 (2009) 123-144.

60. Z. Szymańska, C. M. Rodrigo, M. Łachowicz and M. A. J. Chaplain, Mathematical modelling of cancer invasion of tissue: The role and effect of nonlocal interactions, Math. Models Methods Appl. Sci. 19 (2009) 257-281.

61. Y. Tao, Global existence for a haptotaxis model of cancer invasion with tissue remodeling, Nonlinear Anal. Real World Appl. 12 (2011) 418-435.

62. Y. Tao and M. Winkler, Energy-type estimates and global solvability in a twodimensional chemotaxis-haptotaxis model with remodeling of non-diffusible attractant, J. Differential Equations 257 (2014) 784-815.

63. S. Turner, J. A. Sherratt and D. Cameron, Tamoxifen treatment failure in cancer and the nonlinear dynamics of TGF $\beta$, J. Theor. Biol. 229 (2004) 101-111.

64. A. Van Schepdael, A. Carlier and L. Geris, Sensitivity Analysis by Design of Experiments (Springer, 2016).

65. L. Venkatraman, S.-M. Chia, B. C. Narmada, J. K. White, S. S. Bhowmick, C. F. Dewey, P. T. So, L. Tucker-Kellogg and H. Yu, Plasmin triggers a switchlike decrease in thrombospondin-dependent activation of TGF- $\beta 1$, Biophys. $J$. 103 (2012) 1060-1068.

66. H. Wang, V. Radjendirane, K. K. Wary and S. Chakrabarty, Transforming growth factor $\beta$ regulates cell-cell adhesion through extracellular matrix remodeling and activation of focal adhesion kinase in human colon carcinoma moser cells, Oncogene 23 (2004) 5558-5561. 
67. S. E. Wang, P. Hinow, N. Bryce, A. M. Weaver, L. Estrada, C. L. Arteaga and G. F. Webb, A mathematical model quantifies proliferation and motility effects of TGF- $\beta$ on cancer cells, Comput. Math. Methods Med. 10 (2009) 71-83.

68. J. B. Weitzman, A. Chen and M. E. Hemler, Investigation of the role of $\beta 1$ integrins in cell-cell adhesion, J. Cell Sci. 108 (1995) 3635-3644.

69. M.-S. Wu, J.-T. Lin, P.-N. Hsu, C.-Y. Lin, Y.-T. Hsieh, Y.-H. Chiu, P.-R. Hsueh and K.-W. Liao, Preferential induction of transforming growth factor- $\beta$ production in gastric epithelial cells and monocytes by Helicobacter pylori soluble proteins, J. Infect. Diseases 196 (2007) 1386-1393. 